\title{
A Geometric Method for Determining Intersection Relations between A Movable Convex Object and A Set of Planar Polygons
}

\author{
Kai Tang and Yong-Jin Liu
}

\begin{abstract}
In this paper we investigate how to topologically and geometrically characterize the intersection relations between a movable convex polygon $A$ and a set $\Xi$ of possibly overlapping polygons fixed in the plane. More specifically, a subset $\Phi \subset \Xi$ is called an intersection relation if there exists a placement of $A$ that intersects and only intersects $\Phi$. The objective of this paper is to design an efficient algorithm that finds a finite and discrete representation of all the intersection relations between $A$ and $\Xi$. Past related research only focuses on the complexity of the free space of the configuration space between $A$ and $\Xi$ and how to move or place an object in this free space. However, there are many applications that require the knowledge of not only the free space but also the intersection relations. Examples are presented to demonstrate the rich applications of the formulated problem on intersection relations.
\end{abstract}

Index Terms-Configuration space, intersection relation, geometric and algebraic structure, critical curves and points.

\section{INTRODUCTION}

$\mathrm{I}^{\mathrm{N}}$ this paper, we study a general geometric problem concerning intersection relations between a movable convex polygon and a set of planar polygons. Specifically, let $\mathrm{A}$ be a convex polygon and $\Xi=\left\{\mathrm{P}_{1}, \mathrm{P}_{2}, \ldots, \mathrm{P}_{\mathrm{n}}\right\}$ be a set of $\mathrm{n}$ convex polygons fixed in the plane that can overlap each other. Note that if $\Xi$ contains a concave polygon $P$, we first decompose it into several convex components. Bearing in mind that there exist infinite possible placements of $\mathrm{A}$ in the plane, we present a geometric method to find a finite and discrete representation that encapsulates all the possible intersection relations between $A$ and $\Xi$. Given such a representation, a number of important geometric queries can be readily answered, among them some are:

1) Given a subset of polygons in $\Xi$, is there a placement of $A$ that intersects all of them?

Manuscript received December 5, 2002. This work was supported in part by the Hong Kong University of Science and Technology under Grant DAG02/03.EG33..

Kai Tang is with the Department of Mechanical Engineering, Hong Kong University of Science and Technology, Hong Kong, P.R. China (phone: 852-23588656; fax: 852-23581543; e-mail: mektang@ust.hk).

Yong-Jin Liu is now with the Department of Industrial Engineering and Engineering Management, Hong Kong University of Science and Technology, Hong Kong, P.R. China (e-mail: liuyj@ust.hk).
2) What is the maximal number of polygons that can be intersected by any placement of $\mathrm{A}$ ?

3) When restricted within a required region $D$ in the plane, what is the minimal or maximal number of polygons that can be intersected by a placement of A?

\section{A. Formulation of the Problem}

Consider the general case that the placement of $\mathrm{A}$ is subject to both translations and rotations. Let $\mathrm{g}$ and $\boldsymbol{v}_{\mathrm{g}}$ be a designated reference point and reference vector on A. Any placement of A is thus completely determined by three parameters $(X, Y, \theta)$, where $(X, Y)$ is the position of the reference point $g$ and $\theta$ is the slope angle of vector $\mathbf{v}_{\mathrm{g}}$ in a global coordinate system. Point $(\mathrm{X}, \mathrm{Y}, \theta) \in \mathbf{R}^{2} \times[0,2 \pi)^{1}$ is called a configuration point of $\mathrm{A}$ and the space $\mathrm{X}-\mathrm{Y}-\theta$ is referred to as the configuration space $\mathrm{C}$. To distinguish the configuration space from the object space, we will use capital case X-Y to refer to the configuration plane of the reference point $g$ but small case $x-y$ to represent the object plane in which $A$ and $\Xi$ lie.

A placement of $A$ and a polygon $P \in \Xi$ are said to (1) intersect each other if their interiors intersect, and (2) semi-intersect each other if they only touch each other on their boundaries, and (3) disjoint if they don't share a common point. An intersection relation is a subset $\Phi \subset \Xi$ such that there exists a placement of A, called its realizing placement, that intersects and only intersects $\Phi$. The intersection relation introduces an equivalence relation on the configuration points in $\mathrm{C}$ : two points are said to be equivalent to each other if their corresponding placements of A realize the same intersection relation with $\Xi$. The induced equivalence relation partitions $C$ into a set of equivalent classes which form a quotient space of C.

The set of configuration points of A whose placements intersect $\mathrm{P}$ is known to be a volume, called $C$-primitive in this paper, bounded by patches of ruled surfaces [14]. The C-primitive and its boundary surfaces are denoted by $\mathrm{A}(\mathrm{P})$ and $\mathrm{f}(\mathrm{P})$ respectively. The union $\bigcup_{i=1}^{n} \mathrm{f}\left(\mathrm{P}_{\mathrm{i}}\right)$ subdivides the configuration space into a number of subspaces. These subspaces will be called kernels (cf. Figure 1). Each kernel is represented by its boundary elements, i.e., the boundary faces,

\footnotetext{
${ }^{1}$ Alternatively we may use the stereographic projection to express the orientation $\theta$ of $\mathrm{A}$ in algebraic form.
} 
edges and vertices. Algebraically, each vertex is an intersection point of two edges and each edge is an intersection curve of two ruled surfaces trimmed by its two vertices and each face is a ruled surface trimmed by its boundary edges. We use $\pi(\mathrm{A}, \Xi)$ to represent the union of all the faces on the kernels.

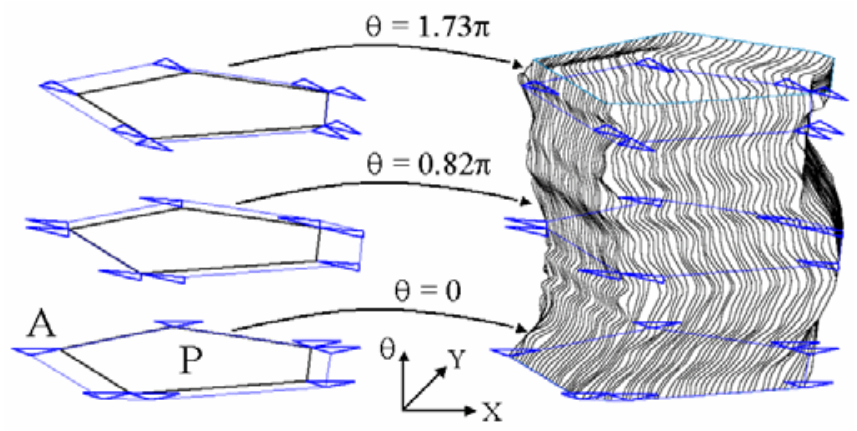

(a) The C-primitive $\mathrm{A}(\mathrm{P})$. The set of configuration points of A whose placements intersect $\mathrm{P}$ is known to be a volume bounded by patches of ruled surfaces in configuration space $\mathrm{X}-\mathrm{Y}-\theta$.

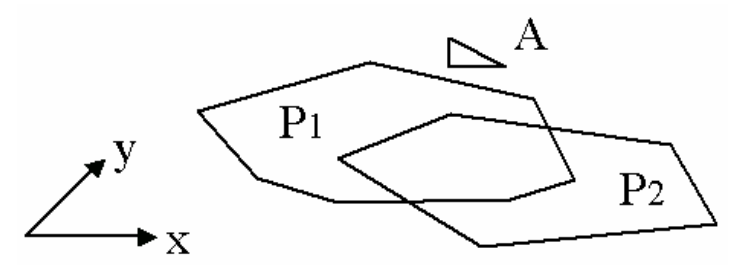

(b) The object space of $\mathrm{A}$ and $\Xi=\left\{\mathrm{P}_{1}, \mathrm{P}_{2}\right\}$

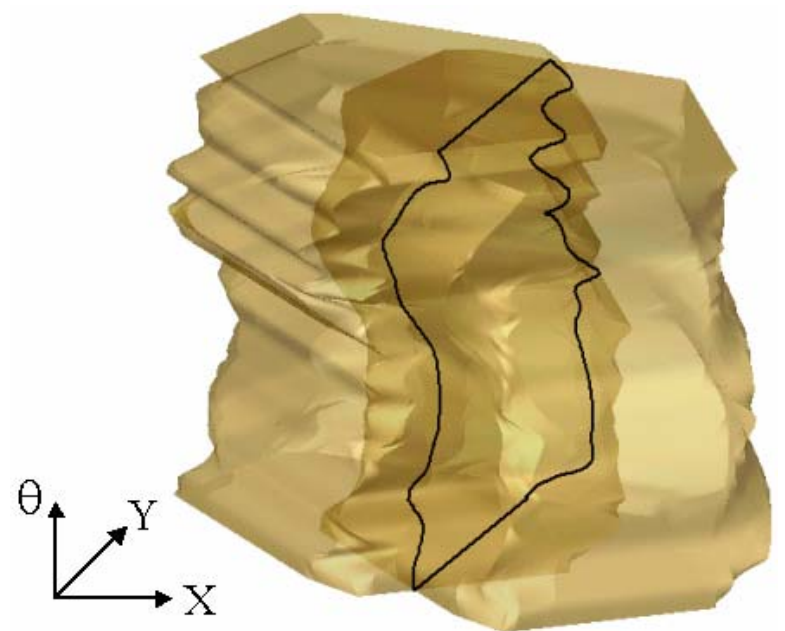

(c) Configuration space decomposition and the kernel representation of the $A$ and $\Xi$ in (b)

Fig. 1. Configuration space decomposition and the kernel representation

The key observation is that, given the induced equivalence relation, the interior of each kernel represents a subset ${ }^{2}$ of an equivalence class. If we pick up one or more representative points from the interior of each kernel, the finite union of these

${ }^{2}$ Since C-primitives are in general concave, an equivalence class may be a union of several kernels. representative points, denoted as $\Sigma^{3}$, is an intersection representative set which satisfies that every intersection relation between $A$ and $\Xi$ has at least one realizing placement in $\Sigma$ and every point in $\Sigma$ is a realizing placement of an intersection relation. For a clear description, the necessary notations used in this paper are summarized in Table I.

The objective of this paper is to find such an intersection representative set $\Sigma$. Rather than directly computing the topological structure of the intersection of C-primitives and building the explicit algebraic structures of kernels, i.e., the algebraic representation of its boundary vertices, edges and faces, we propose a geometric method to obtain an intersection representative set.

TABLE I

SOME NOTATIONS USED IN THIS PAPER

\begin{tabular}{|c|c|}
\hline Symbol & Defintion \\
\hline$\pi(\mathrm{A}, \Xi)$ & The union of all the faces on the kernels \\
\hline$\Sigma$ & An intersection representative set \\
\hline$\omega$ & A contact pair between $A$ and a $P_{i} \in \Xi$ \\
\hline$\sum *{ }^{*}(\omega)$ & A set of points in C that represent the faces in $\pi(\omega)$ \\
\hline$\Sigma^{*}$ & A semi-intersection representative set of $\pi(A, \Xi)$ \\
\hline$E i_{<\mathrm{v}, \mathrm{e}>}\left(\mathrm{t}_{\mathrm{i}}, \mathrm{t}_{\mathrm{i}+1}\right)$ & An eigen set of interval $\left(t_{i}, t_{i+1}\right)$ on e(t) of a $P_{i} \in E$ \\
\hline$C s(<\mathrm{v}, \mathrm{e}>)$ & The characteristic set of contact pair $<v$, e $>$ \\
\hline$E i_{<e, v>}\left(\mathbf{t}_{\mathrm{i}}, \mathrm{t}_{\mathrm{i}+1}\right)$ & An eigen set of interval $\left(t_{i}, t_{i+1}\right)$ on e(t) of $A$ \\
\hline$C s(<\mathrm{e}, \mathrm{v}\rangle)$ & The characteristic set of contact pair $\langle e, v\rangle$ \\
\hline b & The number of edges (or vertices) in $\mathrm{A}$ \\
\hline $\mathbf{n}$ & The number of polygons in $B$ \\
\hline $\mathrm{E}$ & The total number of edges (or vertices) in $\Xi$ \\
\hline
\end{tabular}

\section{B. Related Work and Results of This Paper}

The optimal polygon placement and covering problem [1], [2] has been studied intensively both in robotics research such as motion planning [14], [17], and in manufacturing industries such as material layout [11], facility location decision [4], polygon placement [3], and layer manufacturing [18], [19]. Among them closely related to our work are those concerning the combinatorial complexity of the intersection of $\mathrm{C}$-primitives in the configuration space.

Regarding the configuration space $\mathrm{C}$, previous work focuses on the analysis of a 2-manifold structure, i.e., a topological graph with vertices, edges and faces, on the boundary $\partial \mathrm{FC}$ of the free configuration space FC. Initial results consider only special cases [6]. Often referred to as the Piano Mover's problem I-V [24], Leven and Sharir [16] analyze the combinatorial complexity of $\partial \mathrm{FC}$ using Davenport-Schinzel sequences [22] — the complexity of $\mathrm{FC}$ is shown to be $\mathrm{O}\left(\mathrm{bE} \lambda_{6}(\mathrm{bE})\right)$ and to be $\Omega\left(\mathrm{b}^{2} \mathrm{E}^{2}\right)$ in the worse case, where $\mathrm{b}$ and $\mathrm{E}$ are the total edge numbers of a convex polygon $\mathrm{A}$ and $\mathrm{a}$ polygonal environment, respectively. Later a serial work was proposed to compute FC in a worst-case running time that is near-quadratic in bE [12], [13], [23]. Some most recent significant progresses are proposed in [1], [7]: in [1], a randomized divided-and-conquer algorithm is proposed to compute FC with expected running time $\mathrm{O}\left(\mathrm{bE} \lambda_{6}(\mathrm{bE}) \log (\mathrm{bE}) \log \mathrm{E}\right)$; in [7], the maximum complexity of

\footnotetext{
${ }^{3}$ We refer to a configuration point $\mathrm{q}$ and its placement A(q) interchangeably, unless specially noted in the text.
} 
the free space of a bounded-reach robot with $f$ degrees of freedom, moving in a $\mathbf{R}^{3}$ uncluttered environment, is shown to be $\Theta\left(n^{2 f / 3}+n\right)$, where $n$ is the disjoint polygonal obstacles in the scene.

Although a number of algorithms are proposed in the aforementioned work, they are all targeted to solve certain special geometric problems; none of them can answer all the geometric queries posted in the beginning of this paper. For instance, the optimal convex hull covering problem [2] concerns with finding a specially shaped polygon (e.g., monotone or star shaped) with a minimal area that covers a given set of polygons; however, it does not provide any information about the intersection relations. Similarly, the polygonal placement problem [3] tries to find a feasible (and scaleable) placement of a polygon amid a polygonal environment, which again is incapable of answering any queries related with intersection relations. These intersection relations turn out to be very important in diverse applications concerning geometric optimization, e.g., when combined with the area-of-overlap function [21], the solutions to a number of computer vision problems, such as fuzzy variations of packing [20] and model-based object recognition by probing [9], are readily provided. Another example in the application section of this paper utilizing geographical information is provided to demonstrate the rich applications of intersection relations.

In this paper, by taking an innovative idea based on intrinsic geometric observations, we present an algorithm that finds a representative set $\Sigma$ of the intersection relations between $A$ and $\Xi$. It is shown that the cardinality of $\Sigma$, i.e., the number of points in it, is bounded by $\mathrm{O}(\mathrm{nM})$, where $\mathrm{n}$ is the number of polygons in $B$, and $M$ is the number of critical points that is output-sensitive and has an upper bound $\mathrm{O}\left(\mathrm{b}^{3} \mathrm{E}^{3}\right)$, with $\mathrm{b}$ and $\mathrm{E}$ being the numbers of vertices (or edges) in $A$ and $\Xi$ respectively. The running time required by the proposed algorithm is bounded by $\mathrm{O}\left(\mathrm{b}^{3} \mathrm{E}^{3}+\mathrm{M} \log \mathrm{M}+\mathrm{M}(\mathrm{b} \log \mathrm{b}+\mathrm{bE}+\mathrm{n} \log \mathrm{n})\right)$.

\section{Outline of the Proposed Geometric Method}

Traditionally the model structure in configuration space $\mathrm{C}$ is established via algebraic formulation of $\mathrm{C}$-primitives followed by tedious intersection testing and trimming [10], [27]. To avoid the computationally expensive requirement of algebraic representation, we propose a geometric method to obtain an intersection representative set based on the following geometric observations.

\section{A. Dimension Reduction by Projection Method}

The proposed method partitions $\mathrm{C}$ into a finite collection of algebraic kernels. The 3D kernel can be obtained by computing their projection in lower-dimensional spaces, i.e., its 2D boundary surfaces. Again, the 2D boundary surfaces can be computed by its projection in 1D subspaces, i.e., its boundary curved edges. Geometrically, in object space, the projection can be achieved by enumerating the contact types between A and a polygon $\mathrm{P} \in \Xi$.

Definition 1: A point $(\mathrm{X}, \mathrm{Y}, \theta)$ in the configuration space is a contact configuration point ( $\mathrm{CC}$ for brevity) between $\mathrm{A}$ and a $\mathrm{P} \in \Xi$, if in the object space there exists a point $\mathrm{p} \in \partial \mathrm{A}(\mathrm{X}, \mathrm{Y}$, $\theta) \cap \partial \mathrm{P}$, called a contact point, such that there exists a disk $\Omega$ centering at $\mathrm{p}$ satisfying $\Omega \cap \operatorname{int}(\mathrm{A}(\mathrm{X}, \mathrm{Y}, \theta)) \cap \operatorname{int}(\mathrm{P})=\varnothing$. In addition, a contact (or touch) between $\mathrm{A}(\mathrm{X}, \mathrm{Y}, \theta)$ and $\mathrm{P}$ is said to be of one of the following four contact types:

1) V-E type if a vertex of $A(X, Y, \theta)$ lies on an edge of $P$,

2) $E-V$ type if a vertex of $P$ lies on an edge of $A(X, Y, \theta)$,

3) $\mathrm{V}-\mathrm{V}$ type if a vertex of $\mathrm{A}(\mathrm{X}, \mathrm{Y}, \theta)$ and a vertex of $\mathrm{P}$ coincide, and

4) E-E type if the interior of an edge of $A(X, Y, \theta)$ and the interior of an edge of $\mathrm{P}$ overlap.

The geometric entities of $\mathrm{A}(\mathrm{X}, \mathrm{Y}, \theta)$ and $\mathrm{P}$ that contribute to a contact will be called a contact pair <entity1,entity2>, with the convention that entity 1 and entity 2 belong to $\mathrm{A}$ and $\mathrm{P}$ respectively. For example, if a vertex $\mathrm{v}$ of a placement of $\mathrm{A}$ and an edge e of $\mathrm{P}$ contribute to a V-E contact, then $\langle\mathrm{V}, \mathrm{e}\rangle$ is the contact pair of that contact. Figure 2 illustrates the above-defined types of contact. We further classify a contact pair by its degree.
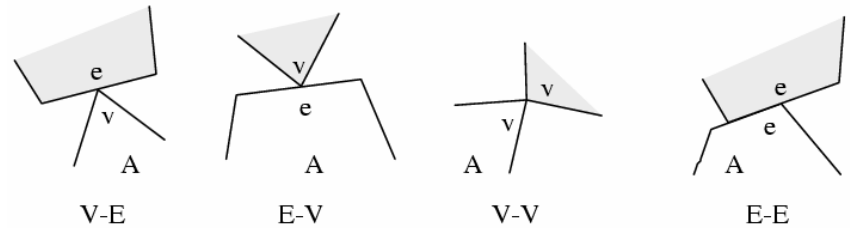

Fig. 2. Contact types

Definition 2: The degree of a contact pair is the number of degrees of freedom of the placement of A when it moves in object space while maintaining the same contact pair.

We note without proof that contact pairs of $\mathrm{V}-\mathrm{E}$ and $\mathrm{E}-\mathrm{V}$ contact types are both of degree two, which form the 2D projection subspaces for the boundary surfaces of kernels in the configuration space, and the degrees of V-V and E-E type contact pairs are both one, which form the 1D projection subspaces in the configuration space.

\section{B. The Perturbation Method}

Consider a contact pair $\omega$ between $A$ and a $P_{i} \in \Xi$. We define three items on $\omega$. The first, to be denoted by $R(\omega)$, is the region in $f\left(P_{i}\right)$ restricted to the contact specified by $\omega$. The second, with a notation of $\pi(\omega)$, is the set of faces in $R(\omega)$ whose edges are intersection curves between $f\left(P_{i}\right)$ and other $f\left(P_{j}\right), i \neq j$, as well as the boundary of $R(\omega)$. The final one is a set $\Sigma^{*}(\omega)$ of points that represent the faces in $\pi(\omega)$, that is, for every face $\phi$ in $\pi(\omega)$, there is at least one point $\xi \in \Sigma^{*}(\omega)$ such that $\xi \in \phi$, and for every point $\xi \in \Sigma^{*}(\omega)$ there is a face $\phi$ in $\pi(\omega)$ such that $\xi \in \phi$. The union $\Sigma^{*}(\mathrm{~A}, \Xi)=\bigcup_{i} \Sigma^{*}\left(\omega_{\mathrm{i}}\right), \Sigma^{*}$ for brevity, over all the possible contact pairs $\omega_{\mathrm{i}}$ between $\mathrm{A}$ and $\Xi$, is obviously a semi-intersection representative set of $\pi(\mathrm{A}, \Xi)$, i.e., $\forall \xi \in \Sigma^{*}$, the placement $\mathrm{A}(\xi)$ semi-intersects a single polygon in $\Xi$ and 
intersects some polygons in $\Xi$, and vice versa.

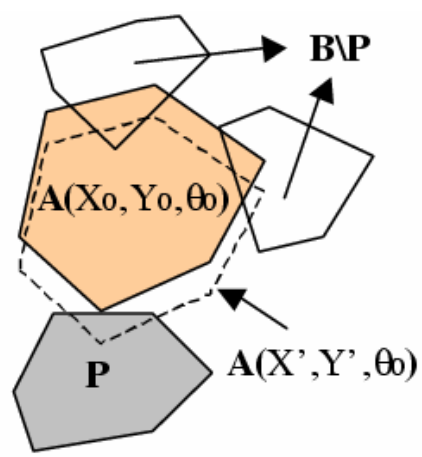

Fig. 3. The perturbation method

As illustrated in Figure 3, given a placement $\mathrm{A}\left(\mathrm{X}_{0}, \mathrm{Y}_{0}, \theta_{0}\right)$ : $\left(\mathrm{X}_{0}, \mathrm{Y}_{0}, \theta_{0}\right) \in \Sigma^{*}$, if we perturb it by translation along the normal between the contact pair by an arbitrarily $( \pm)$ small distance, the resulting configuration point $\left(\mathrm{X}^{\prime}, \mathrm{Y}^{\prime}, \theta_{0}\right)$ is an interior point in each of the two kernels sharing the face containing $\left(X_{0}, Y_{0}, \theta_{0}\right)$. By perturbing each element in $\Sigma^{*}$ in such a way, a superset of intersection representatives with a finite length can be readily obtained. To give a better view of the close relationship between the semi-intersection and intersection representative sets, i.e., $\Sigma^{*}$ and $\Sigma$, Figure 4 depicts an example of a rectangle $\mathrm{A}$ and three polygons $\mathrm{P}_{1}, \mathrm{P}_{2}$, and $\mathrm{P}_{3}$. Set $\left\{\mathrm{A}_{1}, \mathrm{~A}_{2}, \mathrm{~A}_{3}\right\}$ is an intersection representative set, whereas $\left\{\mathrm{B}_{1}, \mathrm{~B}_{2}, \ldots, \mathrm{B}_{6}\right\}$ is a semi-intersection representative set. (For clarity, placements corresponding to a single polygon intersection are not shown.) If we move placement $\mathrm{B}_{5}$, which lies on $\mathrm{f}\left(\mathrm{P}_{1}\right)$, toward $\mathrm{P}_{1}$ by an arbitrarily small distance, it will be an interior point in the kernel represented by placement $\mathrm{A}_{3}$. On the other hand, if we move $\mathrm{B}_{5}$ in the opposite direction away from $\mathrm{P}_{1}$, it falls into another kernel represented $A_{2}$. Now the problem is boiled down to how to find a set $\Sigma^{*}(\omega)$ for a given type of a contact pair $\omega$. As V-V and E-E type are special cases of V-E or E-V type, only $\mathrm{V}-\mathrm{E}$ and $\mathrm{E}-\mathrm{V}$ type need to be considered.
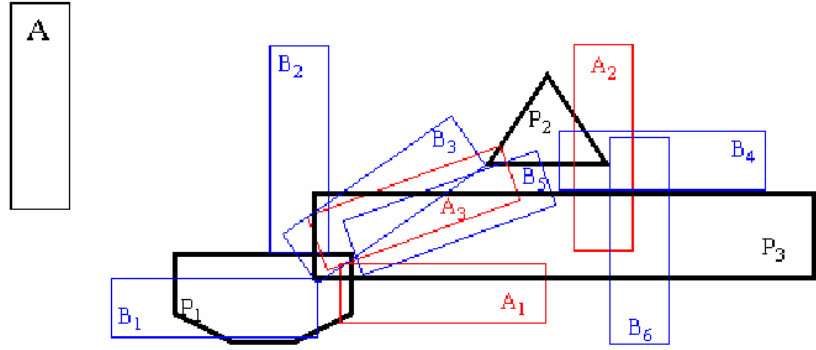

Fig. 4. Relationship between a semi-intersection representative set $\Sigma^{*}$ and an intersection representative set $\Sigma$ (placements corresponding to a single polygon intersection are not shown)

\section{Analysis of Contact Types and Critical Points}

In this section we analyze the algebraic structure of $\pi(A, \Xi)$ and its geometric relation with the critical placements of $\mathrm{A}$ in object space. We use A to refer to the original description of the movable polygon while use $\mathrm{A}(\mathrm{X}, \mathrm{Y}, \theta)$ to represent a particular placement of A.

\section{A. Vertex-Edge Contact Type and Re-parameterization}

Let $\mathrm{v}$ be a vertex on $\mathrm{A}$ and $\mathrm{e}$ be an edge on a $\mathrm{P} \in \Xi$. Consider the region $\mathrm{R}(<\mathrm{v}, \mathrm{e}>)$ on $\mathrm{f}(\mathrm{P})$ attributed to the contact between $\mathrm{v}$ and $\mathrm{e}$. Let $\mathrm{e}(\mathrm{t})$ be a linear parameterization of edge $\mathrm{e}, \mathrm{t} \in[0,1]$, and vertex $\mathrm{v}$ be the reference point $\mathrm{g}$ of $\mathrm{A}$. Since $\mathrm{g}$ is now restricted to be on $\mathrm{e}(\mathrm{t})$ for maintaining the $\mathrm{v}$-e contact, region $\mathrm{R}(<\mathrm{v}, \mathrm{e}>)$ then lies on a "vertical" plane in the $\mathrm{X}-\mathrm{Y}-\theta$ space whose normal is parallel to the X-Y plane. Up to an Euclidean transformation, this plane can be parameterized in configuration space by assigning local coordinates $\mathrm{t}-\theta$ and hence region $\mathrm{R}(<\mathrm{v}, \mathrm{e}>)$ is a continuous region within the rectangle of $[0,1] \times[0,2 \pi)$ in the $t-\theta$ coordinates system (cf. Figure 5). More accurately, region $\mathrm{R}(<\mathrm{v}, \mathrm{e}>$ ) is itself a rectangle bounded by vertical lines $\mathrm{t}=0$ and $\mathrm{t}=1$, and two horizontal lines $\theta=\alpha_{1}$ and $\theta=\alpha_{2}$, where $0<\alpha_{1}<\alpha_{2}<2 \pi$, are determined by the geometry of $\mathrm{A}$ and $\mathrm{P}$. Note that the boundary edges $\mathrm{t}=0$ and $\mathrm{t}=$ 1 correspond respectively to a $\mathrm{V}-\mathrm{V}$ contact with degree one and the boundary edges $\theta=\alpha_{1}$ and $\theta=\alpha_{2}$ each corresponds to an E-E contact also with degree one.

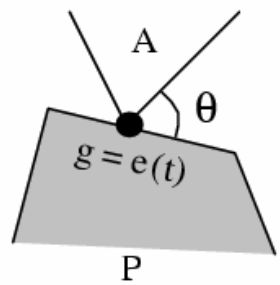

(a) object space

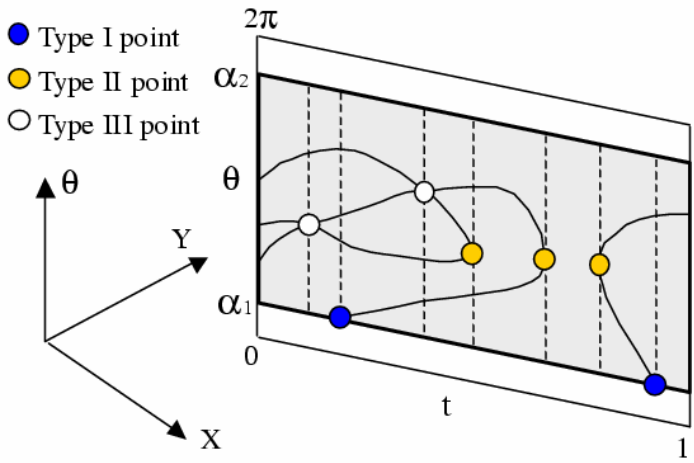

(b) configuration space

Fig. 5. The algebraic structure of the V-E contact type

It is worth noting that if we initially choose a general reference point $g$ and a general reference vector $\mathbf{v}_{\mathrm{g}}$ of $\mathrm{A}$, the region $\mathrm{R}(<\mathrm{V}, \mathrm{e}>)$ is then part of a general (developable) ruled surface; by re-designating the reference point $\mathrm{g}$ from an arbitrary point to vertex $\mathrm{v}$ that is in contact with $\mathrm{P}$, we just re-parameterize the kernel representation in configuration space; in particular, one ruled patch in a kernel boundary is re-parameterized (or equivalently developed) into a planar patch as illustrated in Figure 5(b). This re-parameterization, however, does not change the intersection structure of the 


\section{C-primitives.}

Let $\left\{\chi_{1}, \chi_{2}, \ldots, \chi_{\mathrm{k}}\right\}$ be the intersection curves between $\mathrm{R}(<\mathrm{v}, \mathrm{e}>)$ and all the $\mathrm{f}\left(\mathrm{P}_{\mathrm{i}}\right), \mathrm{P}_{\mathrm{i}} \in \Xi \backslash \mathrm{P}$; they will be called critical curves. The arrangement of these curves, i.e., $\pi(<\mathrm{v}, \mathrm{e}>)$, partitions $\mathrm{R}(<\mathrm{v}, \mathrm{e}>)$ into cells that are themselves or parts of faces of kernels. To avoid explicitly constructing this $\pi(<\mathrm{v}, \mathrm{e}>)$ which requires calculating the intersection curves $\chi_{\mathrm{i}}$ as well as their intersection points, we instead look for a "super" partitioning of this arrangement by super-imposing a trapezoidal decomposition. Let $\left\{\tau_{1}, \tau_{2}, \ldots, \tau_{\mathrm{m}}\right\}$ be the set of the following three types of points (cf. Figure 5(b)):

I) the intersection points between $\chi_{\mathrm{i}}$ and the boundary edge $\theta$ $=\alpha_{1}$ or $\theta=\alpha_{2}$, and

II) the local extreme points of $\chi_{\mathrm{i}}$ in the $\mathrm{t}$ direction, and

III) the intersection points among $\left\{\chi_{\mathrm{i}}\right\}$.

In the following, we first describe how to detect the above three types of points. Then we present how to build up a super partition of $\pi(<\mathrm{v}, \mathrm{e}>)$ with these detected points and their associated eigen and characteristic sets.

(a)

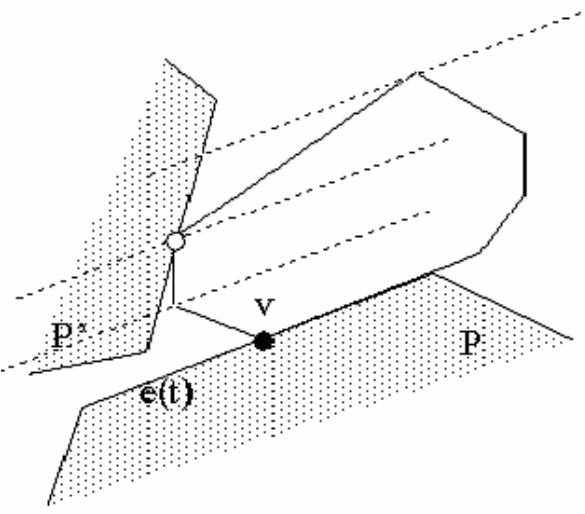

(b)

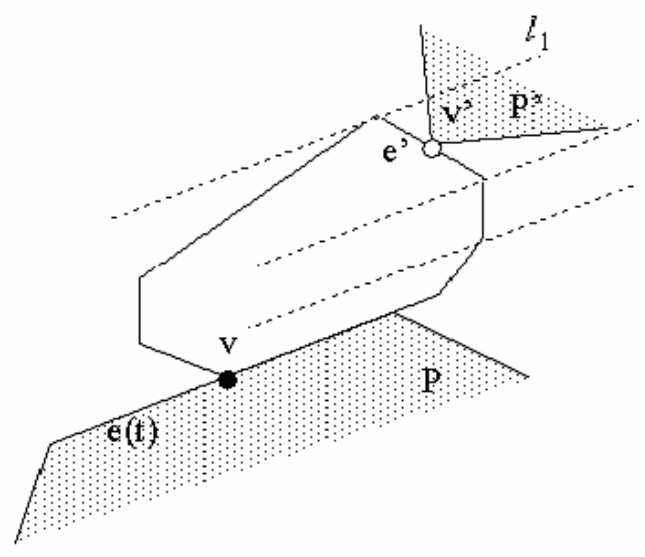

Fig. 6. Type I critical points

\section{1) Type I Critical Points}

This type pertains to the case when a point $\tau_{\mathrm{i}}$ results from the intersection between a curve in $\left\{\chi_{1}, \chi_{2}, \ldots, \chi_{\mathrm{k}}\right\}$ and a boundary line $\theta=\alpha_{1}$ or $\theta=\alpha_{2}$. Geometrically, this means that one of the two edges incident to vertex $\mathrm{v}$ on placement $\mathrm{A}\left(\tau_{\mathrm{i}}\right)$ overlaps with edge e of $\mathrm{P}$ to form a E-E contact and at the same time $\mathrm{A}\left(\tau_{\mathrm{i}}\right)$ also touches another polygon $\mathrm{P}^{\prime} \in \Xi \backslash \mathrm{P}$. As revealed in Figure 6, disregarding degenerate cases, the contact between $\mathrm{A}\left(\tau_{\mathrm{i}}\right)$ and $\mathrm{P}^{\prime}$ can be either a V-E contact (cf. Figure 6(a)) or an E-V contact (cf. Figure 6(b)). Figure 6 also demonstrates how a Type I point should be identified. Depending on which boundary line of $\theta=\alpha_{1}$ or $\theta=\alpha_{2}$ to check, A is placed by overlapping one of its two edges incident to $v$ with edge $e(t)$. We then draw lines parallel to $\mathrm{e}(\mathrm{t})$ through each vertex of the this placement. For example, as shown in Figure 6(b), to check if vertex v' on P' contributes to a Type I critical point together with the prescribed E-E contact, parallel lines $l_{1}, l_{2}$, and $l_{3}$ are drawn; if one of the stripes between $l_{1}, l_{2}$, and $l_{3}$ encloses v', v' may then contribute to a Type I critical point and further test utilizing distance measure is applied. Similar arguments can be made for calculating a Type I critical point with V-E and E-E contact type, as shown in Figure 6(a).

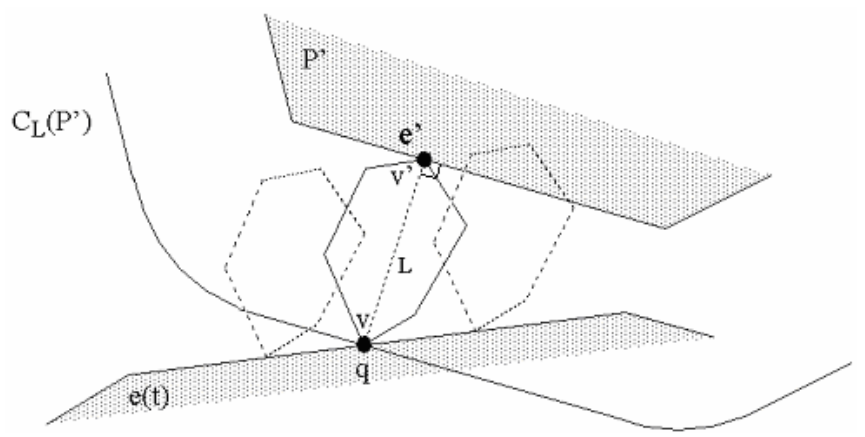

Fig. 7. Type II critical points

\section{2) Type II Critical Points}

A point $\tau_{\mathrm{i}}$ on a curve $\chi \in\left\{\chi_{1}, \chi_{2}, \ldots, \chi_{\mathrm{k}}\right\}$ is a t-extreme point if $\chi$ is locally supported by the vertical line $\mathrm{t}=\mathrm{t}_{\mathrm{i}}$ that is tangent to $\chi$ at $\tau_{\mathrm{i}}$. Suppose $\chi$ is contributed by $\mathrm{P}$ and one other polygon $\mathrm{P}^{\prime} \in \Xi \backslash \mathrm{P}$. Being a t-extreme point means that there exist a $\Delta \mathrm{t}>0$ such that for any $t \in\left(t_{i}-\Delta t, t_{i}\right)$ there is a $\theta \in\left(\alpha_{1}, \alpha_{2}\right)$ such that the placement $\mathrm{A}(\mathrm{e}(\mathrm{t}), \theta)$ intersects $\mathrm{P}$ ' whereas no such pair $(\mathrm{t}, \theta)$ can be found in the interval $t \in\left(t_{i}, t_{i}+\Delta t\right)$, or vice versa. To characterize this extremeness geometrically, as illustrated in Figure 7, let $\mathrm{L}$ be the largest distance between vertex $\mathrm{v}$ and any other vertices on $A$, say between $v$ and v', and $C_{L}\left(P^{\prime}\right)$ be the

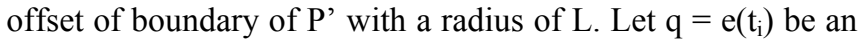
intersection point between $\mathrm{C}_{\mathrm{L}}\left(\mathrm{P}^{\prime}\right)$ and edge $\mathrm{e}(\mathrm{t})$, if such an intersection exists. Without loss of generality, suppose edge e(t) goes "into" $C_{L}\left(P^{\prime}\right)$ at $t_{i}$; that is, there is a $\Delta t>0$ such that $\mathrm{e}(\mathrm{t}): \mathrm{t} \in\left(\mathrm{t}_{\mathrm{i}}-\Delta \mathrm{t}, \mathrm{t}_{\mathrm{i}}\right)$ are outside $\mathrm{C}_{\mathrm{L}}\left(\mathrm{P}^{\prime}\right)$ whereas $\mathrm{e}(\mathrm{t}): \mathrm{t} \in\left(\mathrm{t}_{\mathrm{i}}, \mathrm{t}_{\mathrm{i}}+\Delta \mathrm{t}\right)$ are inside $C_{L}\left(P^{\prime}\right)$. Obviously, for any point $t \in\left(t_{i}-\Delta t, t_{i}\right), A(e(t), \theta)$ can not intersect $P^{\prime}$ for any $\theta$, but for any $t \in\left(t_{i}, t_{i}+\Delta t\right)$ there must exist a placement of A for some $\theta$ ' that intersects $\mathrm{P}$ '. To ensure that $t_{i}$ is not a "false" critical point, however, $\theta$ ' should be in the interval of $\left(\alpha_{1}, \alpha_{2}\right)$. This condition can be easily examined by first locating the unique placement $\mathrm{A}\left(\mathrm{e}\left(\mathrm{t}_{\mathrm{i}}\right), \theta^{\prime}\right)$ that touches $\mathrm{P}^{\prime}$ at its vertex $\mathrm{v}^{\prime}$ ' and then verifying if the angle $\theta$ ' is in the range $\left(\alpha_{1}, \alpha_{2}\right)$. Note that in the placement $\mathrm{A}\left(\mathrm{e}\left(\mathrm{t}_{\mathrm{i}}\right), \theta^{\prime}\right)$, the line segment $\mathrm{vv}^{\prime}$ is perpendicular to the edge e' of $\mathrm{P}^{\prime}$ that is in contact with v' of A.

3) Type III Critical Points

This third type refers to a point $\tau_{\mathrm{i}}$ that is the intersection 
between two different curves in $\left\{\chi_{1}, \chi_{2}, \ldots, \chi_{\mathrm{k}}\right\}$. Suppose $\chi^{\prime}$ and $\chi$ ' are contributed by some $\mathrm{P}$ ' and $\mathrm{P}$ " from $\left\{\mathrm{P}_{1}, \mathrm{P}_{2}, \ldots, \mathrm{P}_{\mathrm{n}}\right\}$ respectively. Their intersection point $\tau_{\mathrm{i}}$ is easily seen to be a "corner" on some kernels - it is the intersection between three surfaces $\mathrm{f}(\mathrm{P}), \mathrm{f}\left(\mathrm{P}^{\prime}\right)$, and $\mathrm{f}\left(\mathrm{P}^{\prime}\right)$. Thus, the placement $\mathrm{A}\left(\tau_{\mathrm{i}}\right)$ touches $\mathrm{P}, \mathrm{P}$ ' and $\mathrm{P}$ ' simultaneously, with a constraint that the contact pair between $\mathrm{A}\left(\tau_{\mathrm{i}}\right)$ and $\mathrm{P}$ must be $<\mathrm{v}, \mathrm{e}>$.

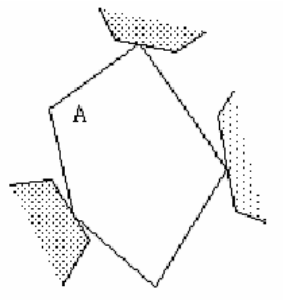

V-E, V-E, V-E

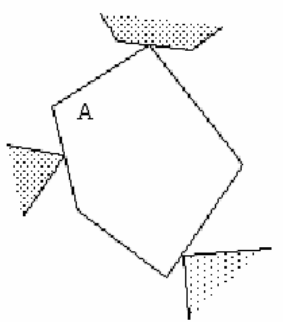

V-E, E-V, E-V

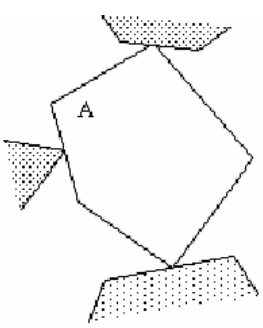

V-E, V-E, E-V

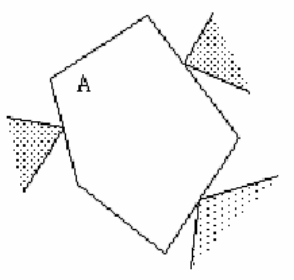

E-V, E-V, E-V
Fig. 8. Triple-contact placements

A placement of A that touches three different polygons in $\Xi$ will be called a triple-contact placement. Figure 8 depicts all the possible four cases of triple-contact placement based on the types of their contact pairs, namely $\{\mathrm{V}-\mathrm{E}, \mathrm{V}-\mathrm{E}, \mathrm{V}-\mathrm{E}\}$, $\{\mathrm{V}-\mathrm{E}, \mathrm{V}-\mathrm{E}, \mathrm{E}-\mathrm{V}\},\{\mathrm{V}-\mathrm{E}, \mathrm{E}-\mathrm{V}, \mathrm{E}-\mathrm{V}\}$, and $\{\mathrm{E}-\mathrm{V}, \mathrm{E}-\mathrm{V}, \mathrm{E}-\mathrm{V}\}$, from which the first three cases may contribute to a Type III critical point with the constraint to maintain a V-E contact pair $<\mathrm{V}, \mathrm{e}>$ between $\mathrm{A}$ and $\mathrm{P}$.

To calculate the configuration point for each triple-contact placement, from each two of three contact pairs one critical curve is formed and the configuration point is the intersection of any two such critical curves, if it exists. These critical curves, belonging to the general family of glissettes [15], are all algebraic and therefore smooth except at finite singular points. In particular, the critical curves resulted from $\{(\mathrm{V}-\mathrm{E}),(\mathrm{V}-\mathrm{E})\}$ and $\{(\mathrm{E}-\mathrm{V}),(\mathrm{E}-\mathrm{V})\}$ are portions of ellipses, and the critical curve resulted from $\{(\mathrm{V}-\mathrm{E}),(\mathrm{E}-\mathrm{V})$ is portion of a quadratic curve. We present a detailed description of these algebraic curves in parametric form in Appendix.

4) Angular Partitioning, Eigen and Characteristic Set

With respect to a vertex $v$ on $A$, once all the critical points on edge $\mathrm{e}(\mathrm{t})$ of $\mathrm{P}$ are detected, in terms of local parameter $\mathrm{t}$, we collect and sort them into a list $\left\{\mathrm{t}_{1}, \mathrm{t}_{2}, \ldots, \mathrm{t}_{\mathrm{m}}\right\}$. Given the sequence $\left\{\mathrm{t}_{0}=0, \mathrm{t}_{1}, \mathrm{t}_{2}, \ldots, \mathrm{t}_{\mathrm{m}}, \mathrm{t}_{\mathrm{m}+1}=1\right\}$, we pick up an arbitrary point $\mathrm{q} \in\left(\mathrm{t}_{\mathrm{i}}, \mathrm{t}_{\mathrm{i}+1}\right)$ for each interval, $\mathrm{i}=0,1, \ldots, \mathrm{m}$. As illustrated in Figure 9, the intersection between the vertical line $\mathrm{t}=\mathrm{q}$ and critical curves $\left\{\chi_{1}, \chi_{2}, \ldots, \chi_{\mathrm{k}}\right\}$ in $\mathrm{R}(<\mathrm{v}, \mathrm{e}>)$ results in a list of collinear points in the X-Y- $\theta$ space $\left\{\left(\mathrm{q}, \theta_{1},\right),\left(\mathrm{q}, \theta_{2}\right), \ldots,\left(\mathrm{q}, \theta_{\mathrm{h}}\right)\right\}$. Conceivably, any placement $\mathrm{A}\left(\mathrm{q}, \theta_{\mathrm{i}}\right)$ has a contact pair $<\mathrm{v}, \mathrm{e}>$ with $\mathrm{P}$ and at the same time touches some other polygon $\mathrm{P}_{\mathrm{i}} \in \Xi \backslash \mathrm{P}$. Assuming $\theta_{1}, \theta_{2}, \ldots, \theta_{\mathrm{h}}$ are already sorted in ascending order, the ordered list $\left\{\theta_{0}=\alpha_{1}, \theta_{1}, \theta_{2}, \ldots, \theta_{\mathrm{h}}, \theta_{\mathrm{h}+1}=\alpha_{2}\right\}$ will be defined as the angular partitioning at point q. Let $\xi_{\mathrm{i}}$ be an arbitrary number in interval $\left(\theta_{i}, \theta_{i+1}\right), i=0,1, \ldots, h$, the set $\{(q$, $\left.\left.\xi_{0}\right),\left(\mathrm{q}, \xi_{1}\right), \ldots,\left(\mathrm{q}, \xi_{\mathrm{h}}\right)\right\}$ is called an eigen set of interval $\left(\mathrm{t}_{\mathrm{i}}, \mathrm{t}_{\mathrm{i}+1}\right)$ on e(t), to be denoted as $E i_{<\mathrm{v}, \mathrm{e}}\left(\mathrm{t}_{\mathrm{i}}, \mathrm{t}_{\mathrm{i}+1}\right)$. In Figure 10, an example illustrating angular partitioning and eigen set in object space is presented. Equipped with angular partitioning and eigen sets, we define the characteristic set of contact pair $\langle\mathrm{v}$, e $>$ to be the union of the eigen sets on $\mathrm{e}(\mathrm{t})$, i.e., $\operatorname{Cs}(<\mathrm{v}, \mathrm{e}>)=\bigcup_{i} E i_{<\mathrm{v}, \mathrm{e}>}\left(\mathrm{t}_{\mathrm{i}}\right.$, $\left.t_{i+1}\right)$. We present the following lemma with its proof omitted.

Lemma 1: The characteristic set $C s(<\mathrm{v}, \mathrm{e}>)$ is a superset of the representative points of $\pi(<\mathrm{v}, \mathrm{e}>)$. That is, every face $\phi$ in $\pi(\mathrm{v}, \mathrm{e})$ has at least one corresponding point $\xi$ in $C s(<\mathrm{v}, \mathrm{e}>)$ such that $\xi \in \phi$, and for every point $\xi \in C s(<\mathrm{v}, \mathrm{e}>)$ there is a face $\phi$ in $\pi$ $(<\mathrm{v}, \mathrm{e}>)$ such that $\xi \in \phi$.

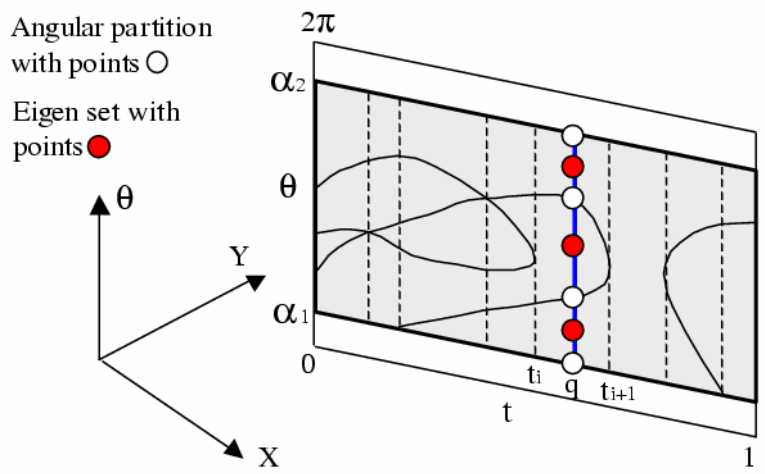

Fig. 9. Angular partitioning and eigen set of $\mathrm{q}$ in configuration space

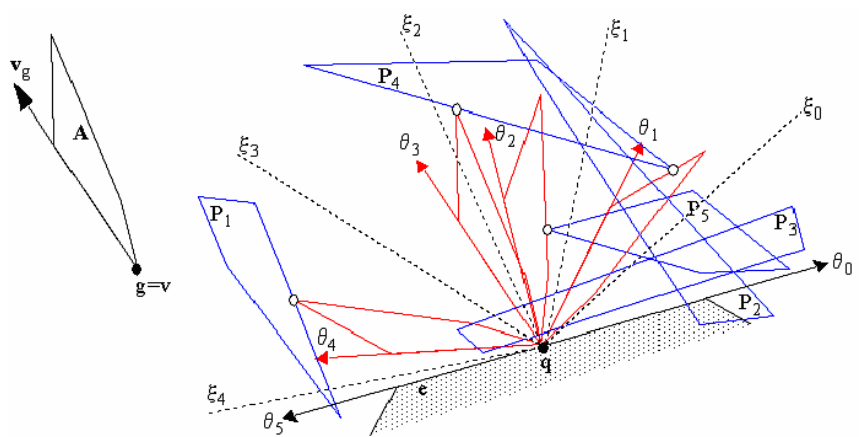

Fig. 10. Angular partitioning and eigen set of $\mathrm{q}$ in object space

\section{B. Edge-Vertex Contact Type}

Having found a representative set $\Sigma^{*}(\omega)$ for the $\mathrm{V}$-E contact type, we now proceed to search for a representative set for the E-V contact type. First of all, it is important to state the necessity of this task. In terms of intersection relations, it is conceivable that not every point in a representative set of $\pi(\mathrm{A}$, $\Xi)$ is equivalent to a $C C$ point attributed to a V-E type contact. For instance, in the example given in Figure 11, it can be easily 
verified that all the placements of $\mathrm{A}$ that touch $\mathrm{P}$ and intersect P' only must have an E-V contact with $\mathrm{P}$.

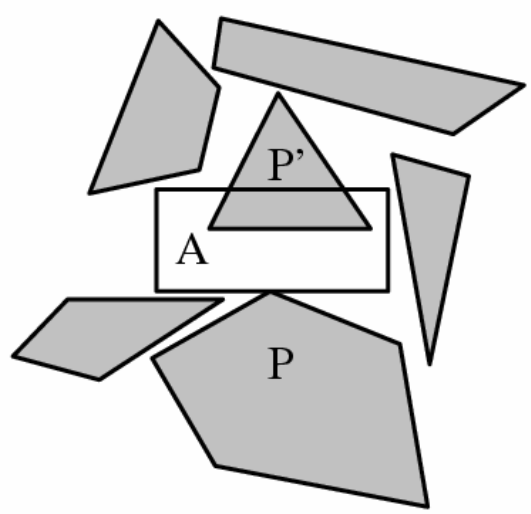

Fig. 11. A placement of A that touches $P$ with an E-V type contact and intersects a single polygon $\mathrm{P}^{\prime}$

Let $e(t)(t \in[0,1])$ be the linear parametric representation of an edge $\mathrm{e}$ of $\mathrm{A}$ that is required to contact a vertex $\mathrm{v}$ on $\mathrm{a}$ polygon $\mathrm{P} \in \Xi$. If we choose one of two vertices adjoining to edge $\mathrm{e}$ of $\mathrm{A}$ as the reference point and re-parameterize the kernel representation, the region $\mathrm{R}(<\mathrm{e}, \mathrm{v}>)$ is then part of a helicoid, the only ruled minimal surface other than the plane [8]. Like in the case of the V-E type, a similar analysis of the algebraic structure can be conducted on this helicoid surface. For a clear and concise representation, we though go directly to take a geometric approach to find a representative set of the faces in $\pi(<\mathrm{e}, \mathrm{v}>)$, based on the concept of the contact order and its induced congruence relation. Suppose A contacts $v$ of $P$ at a point $e(t)$ on edge e for some $t$. Imagine A is "hinged" at the contact $v-e(t)$ and can rotate about this hinge point; let $A_{t}(\theta)$ represent the placement of this hinged $A$ that has an orientation $\theta$. Let $\left\{\mathrm{A}_{\mathrm{t}}\left(\theta_{1}\right), \mathrm{A}_{\mathrm{t}}\left(\theta_{2}\right), \ldots, \mathrm{A}_{\mathrm{t}}\left(\theta_{\mathrm{h}}\right)\right\}$ be the placements with each of them touching a different polygon in $\Xi$ (in addition to $P$ ), and $\left\{\omega_{1}, \omega_{2}, \ldots, \omega_{\mathrm{h}}\right\}$ be the corresponding contact pairs. Assuming these $\theta_{\mathrm{i}}$ are already sorted in ascending order, the ordered list $<\omega_{1}, \omega_{2}, \ldots, \omega_{\mathrm{h}}>$ is defined to be the contact order of point $\mathrm{e}(\mathrm{t})$, denoted as $\operatorname{Co}(\mathrm{t})$. An example is given in Figure 12.

Let $\theta_{0}=\alpha_{1}$ and $\theta_{\mathrm{h}+1}=\alpha_{2}$, where $\alpha_{1}$ and $\alpha_{2}$ are respectively the critical orientations of the two placements of A when its edge $\mathrm{e}$ in contact overlaps with each of the two edges of $\mathrm{P}$ incident to vertex $\mathrm{v}$. It is straightforward to see that, for every $\mathrm{i}$ $=0,1, \ldots, \mathrm{h}$, (the configuration points of) all the placements $\mathrm{A}_{\mathrm{t}}(\theta): \theta \in\left(\theta_{\mathrm{i}}, \theta_{\mathrm{i}+1}\right)$ are in a same face in $\pi(<\mathrm{e}, \mathrm{v}>)$, and thus any one of them, say $\mathrm{A}_{\mathrm{t}}\left(\xi_{\mathrm{i}}\right), \quad \xi_{\mathrm{i}} \in\left(\theta_{\mathrm{i}}, \theta_{\mathrm{i}+1}\right)$, can serve as a representative point of that face. The list $f l(t)=\left\{\pi^{-1}\left(\xi_{0}\right), \pi^{-1}\left(\xi_{1}\right)\right.$, $\left.\ldots, \pi^{-1}\left(\xi_{\mathrm{h}}\right)\right\}$ is then called the face list at $\mathrm{t}$, where we use $\pi^{-1}\left(\xi_{\mathrm{i}}\right)$ to denote the face in $\pi(<\mathrm{e}, \mathrm{v}>)$ represented by $\mathrm{A}_{\mathrm{t}}\left(\xi_{\mathrm{i}}\right)$. The set of placements $\left\{A_{t}\left(\xi_{0}\right), A_{t}\left(\xi_{1}\right), \ldots, A_{t}\left(\xi_{h}\right)\right\}$ is thus a representative set of $f l(\mathrm{t})$.

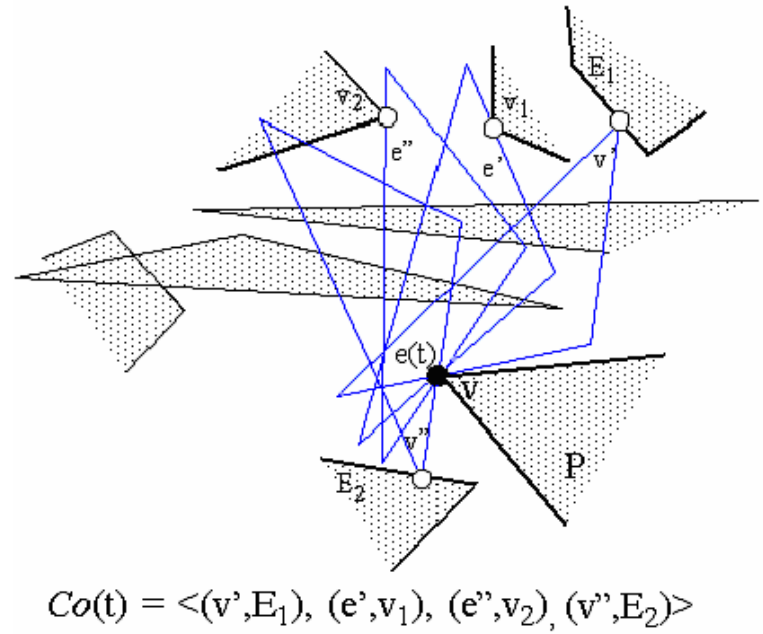

Fig. 12. Contact order of point $\mathrm{e}(\mathrm{t})$ with respect to vertex $\mathrm{v}$

To find a representative set of the faces in $\pi(<\mathrm{e}, \mathrm{v}>)$ geometrically, the strategy is to partition edge $e(t)$ into a set of intervals such that within each interval all the points have the same face list. To be more specific, an interval $\mathrm{I} \subset[0,1]$ is called a congruent interval if all the contact orders $C o(t): t \in I$ are identical to each other. It can be shown that all the points in a congruent interval have the same face list. Let $0=\mathrm{t}_{0}<\mathrm{t}_{1}<\mathrm{t}_{2}<\ldots<\mathrm{t}_{\mathrm{m}}<\mathrm{t}_{\mathrm{m}+1}=1$ be $\mathrm{m}$ such critical points that partition $e(t)$ into $m+1$ congruent intervals $\left(t_{i}, t_{i+1}\right)$. Akin to the vertex-edge contact type case, we define an eigen set of interval $\left(t_{i}, t_{i+1}\right)$, denoted by $E i_{<e, v>}\left(t_{i}, t_{i+1}\right)$, as the representative set of a face list $f l\left(\tau_{\mathrm{i}}\right)$ where $\tau_{\mathrm{i}}$ can be any arbitrary number in $\left(\mathrm{t}_{\mathrm{i}}, \mathrm{t}_{\mathrm{i}+1}\right)$. The characteristic set of contact pair $<\mathrm{e}, \mathrm{v}\rangle$ is then defined to be the union of all the eigen sets, that is, $\operatorname{Cs}(<\mathrm{e}, \mathrm{v}>)=\bigcup_{i=0}^{m} E i_{<\mathrm{e}, \mathrm{v}>}\left(\mathrm{t}_{\mathrm{i}}\right.$, $t_{i+1}$ ). Therefore, similar to lemma 1 , we have the following lemma.

Lemma 2: The characteristic set $\operatorname{Cs}(<\mathrm{e}, \mathrm{v}>)$ is a superset of the representative points of $\pi(<\mathrm{e}, \mathrm{v}>)$. That is, every face $\phi$ in $\pi(<\mathrm{e}, \mathrm{v}>)$ has at least one corresponding point $\xi$ in $\operatorname{Cs}(<\mathrm{e}, \mathrm{v}>)$ such that $\xi \in \phi$, and for every point $\xi \in C s(<\mathrm{e}, \mathrm{v}>)$ there is a face $\phi$ in $\pi(<\mathrm{e}, \mathrm{v}>)$ such that $\xi \in \phi$.

Just how should this congruent partitioning be achieved? Notice that for a small perturbation $\delta, C o(t)$ should remain unchanged, i.e., $\operatorname{Co}(\mathrm{t}+\delta)=\operatorname{Co}(\mathrm{t})$, so should their face list. This suggests that we only need to look at a t where the contact order changes. Calling such a t a critical point, we next characterize it geometrically in terms of the conditions causing the change.

1) Type IV-Emerging and Disappearing of A Contact Pair

Given $t_{1}, t_{2} \in[0,1]$, to determine whether or not two contact orders $C o\left(t_{1}\right)$ and $C o\left(t_{2}\right)$ are identical to each other, the first necessary condition is that the number of contact pairs in both are the same. Suppose $\omega_{\mathrm{i}}$ is a contact pair between $\mathrm{A}$ and $\mathrm{P}_{\mathrm{i}}$ that is in $\mathrm{Co}\left(\mathrm{t}_{1}\right)$ but no longer exists in $\operatorname{Co}\left(\mathrm{t}_{2}\right)$. Because the contact point between $A$ and $P_{i}$ associated with $\omega_{i}$ moves continuously along the boundary of $\mathrm{P}_{\mathrm{i}}$ or $\mathrm{A}$ (depending on the contact type of $\omega_{i}$ ) when $t$ changes from $t_{1}$ to $t_{2}$, there must exist a critical point $t^{\prime} \in\left(t_{1}, t_{2}\right)$ such that $\omega_{i}$ is in all of $\operatorname{Co}(t): t \in\left(t_{1}, t^{\prime}\right)$ but in none of 
$\operatorname{Co}(\mathrm{t}): \mathrm{t} \in\left(\mathrm{t}^{\prime}, \mathrm{t}_{2}\right)$. There can only be two possible causes of this disappearing:

i) The first is that, although at $t_{2}$ there is still a placement $A_{t 2}(\theta)$ that touches $P_{i}$, the orientation $\theta$ is nonetheless outside the range $\left(\alpha_{1}, \alpha_{2}\right)$, and consequently $<\mathrm{e}, \mathrm{v}>$ is not a contact pair between $A_{t 2}(\theta)$ and $P$ (cf. Figure 13). The critical point t' due to this case is categorized to be a Type IVa point.

ii) The second scenario is that no placement $A_{t 2}(\theta)$ at $t_{2}$ can be found for any angle $\theta$ that touches $\mathrm{P}_{\mathrm{i}}$, and the critical point $\mathrm{t}$ ' contributing to this case is counted as a Type IVb point (cf. Figure 14).
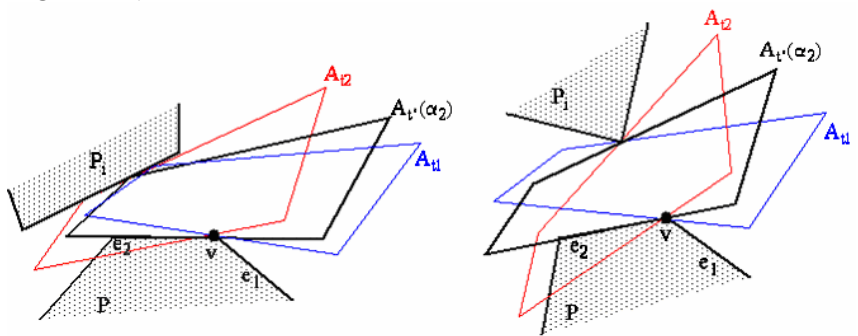

Fig. 13. Type IVa critical points

Figure 13 depicts two examples illustrating the first case, each for a V-E and an E-V contact between $\mathrm{P}_{\mathrm{i}}$ and $\mathrm{A}$, respectively. It also demonstrates how a Type IVa point should be calculated on edge e - we simply "slide" A with a fixed orientation $\theta=\alpha_{1}$ (resp. $\theta=\alpha_{2}$ ) on its edge e along the line of edge $\mathrm{e}_{1}\left(\right.$ resp. $\mathrm{e}_{2}$ ) of $\mathrm{P}$ until it is in touch with $\mathrm{P}_{\mathrm{i}}$, at which the point $\mathrm{e}(\mathrm{t})$ that coincides with vertex $\mathrm{v}$, if it exists, is a Type IVa critical point on edge e of A. It is worth mentioning that Type IVa critical points are identical to Type I critical points, if we interpret the degree-1 E-E contact type as a composition of two degree-2 contact types E-V and V-E.
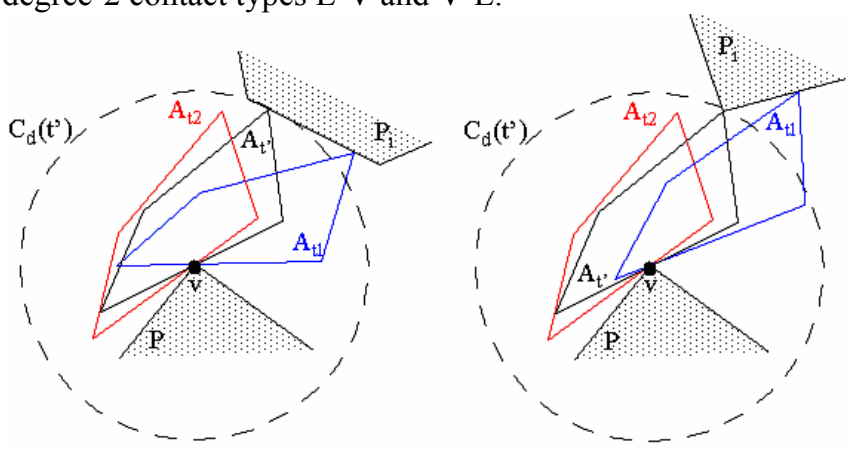

Fig. 14. Type IVb critical points

To geometrically characterize a Type IVb point, intuitively, we should look for a t' where a placement $\mathrm{A}_{\mathrm{t}},(\theta)$ is in "tangent contact" with $\mathrm{P}_{\mathrm{i}}$. To describe this tangent contact more rigorously, the concepts of covering radius and covering disc are introduced. For a $t \in[0,1]$, the covering radius $\mathrm{R}_{\mathrm{c}}(\mathrm{t})$ is defined to be $\max \{\|\mathrm{e}(\mathrm{t})-\mathrm{p}\|: \mathrm{p} \in \mathrm{A}\}$. Noting that since $\mathrm{A}$ is a polygon, $R_{c}(t)$ is actually the largest distance from point $e(t)$ in $A$ to any vertex of $A$. The covering $\operatorname{disc} C_{d}(t)$ is then the circular disc of radius $R_{c}(t)$ with the center at the contact vertex $v$ of $P$. Referring to Figure 14, if $\mathrm{P}_{\mathrm{i}}$ touches a covering disc $\mathrm{C}_{\mathrm{d}}\left(\mathrm{t}^{\prime}\right)$ for some $t^{\prime} \in[0,1]$, disregarding the degenerate cases, $t$ ' is certainly a Type IVb point. The converse of this assertion can also be proven easily. Therefore, a t' is a Type IVb point if and only if there is some polygon $P_{i} \in \Xi$ that touches the covering disc $\mathrm{C}_{\mathrm{d}}\left(\mathrm{t}^{\prime}\right)$.

Figure 15 shows how a Type IVb point should be calculated. For any vertex v' or edge e' on any polygon $\mathrm{P}_{\mathrm{i}} \in \Xi\left(\mathrm{P}_{\mathrm{i}} \neq \mathrm{P}\right)$, we first check if $P_{i}$ touches the disc $C_{L}$ that centers at $v$ of $P$ and has a radius $\mathrm{L}$ equal to the distance from $\mathrm{v}$ to $\mathrm{v}$ ' or e' (cf. Figure 15(a)). If this is true, as exemplified in Figure 15(b), we then with every vertex on $\mathrm{A}$ as a center draw a circle of radius $\mathrm{L}$ to intersect edge e of $\mathrm{A}$. If the intersection exists, we check whether the circle $C_{L}$ of radius $L$ centering at the intersection point encloses $\mathrm{A}$. If this is true, the intersection point is reported as a Type IVb critical point (e.g., in the example shown in Figure $15(b), t_{2}$ is a Type IVb point but $t_{1}$ is not).
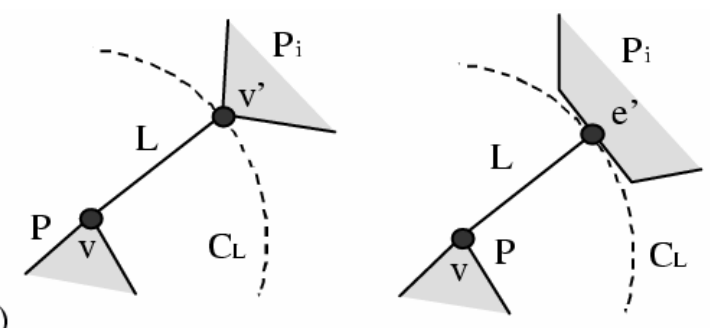

(a)

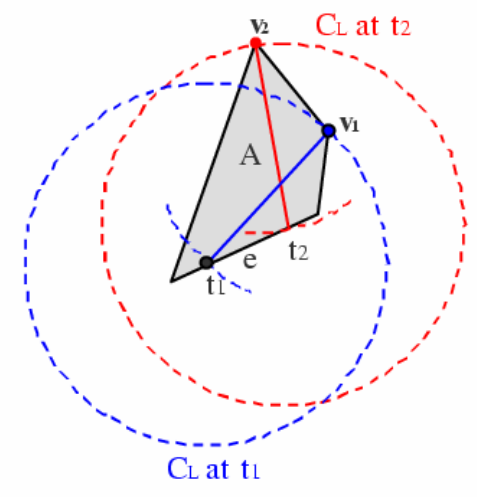

Fig. 15. The conditions for checking the existence of Type IVb critical points

2) Type V-Order Switching of Two Contact Pairs

Suppose now we are assured that an interval $\left[\mathrm{t}_{1}, \mathrm{t}_{2}\right] \subset[0,1]$ is free of Type IV critical points. As a result, all the contact orders $\operatorname{Co}(\mathrm{t}): \mathrm{t} \in\left[\mathrm{t}_{1}, \mathrm{t}_{2}\right]$ should have the same set of contact pairs. Let $\operatorname{Co}\left(\mathrm{t}_{1}\right)=<\omega_{1}, \omega_{2}, \ldots, \omega_{\mathrm{h}}>$. If $\operatorname{Co}\left(\mathrm{t}_{2}\right)$ is different from $\operatorname{Co}\left(\mathrm{t}_{1}\right)$, there must be a contact pair $\omega_{i}$ between $\mathrm{A}$ and a $\mathrm{P}$ ' whose position (or index) in $\operatorname{Co}\left(\mathrm{t}_{2}\right)$ is different from i. Let $\theta_{\mathrm{i}}(\mathrm{t}), \mathrm{t} \in\left[\mathrm{t}_{1}, \mathrm{t}_{2}\right]$, be the orientation of the placement of $\mathrm{A}$ associated with $\omega_{i}$; that is, $\mathrm{A}_{\mathrm{t}}\left(\theta_{\mathrm{i}}(\mathrm{t})\right)$ touches $\mathrm{P}^{\prime}$ at $\omega_{\mathrm{i}}$. It is trivial to see that for all $\mathrm{i}, \theta_{\mathrm{i}}(\mathrm{t})$ is a continuous function of $\mathrm{t}$ in $\left[\mathrm{t}_{1}, \mathrm{t}_{2}\right]$ (cf. Figure 13). If $\omega_{\mathrm{i}}$ undergoes an index change from $\operatorname{Co}\left(\mathrm{t}_{1}\right)$ to $C o\left(\mathrm{t}_{2}\right)$, there must exist a $\mathrm{t}^{\prime} \in\left(\mathrm{t}_{1}, \mathrm{t}_{2}\right)$ where either $\theta_{\mathrm{i}}\left(\mathrm{t}^{\prime}\right)=\theta_{\mathrm{i}+1}\left(\mathrm{t}^{\prime}\right)$ or $\theta_{\mathrm{i}}\left(\mathrm{t}^{\prime}\right)=\theta_{\mathrm{i}-1}\left(\mathrm{t}^{\prime}\right)$. This implies however that the placement $\mathrm{A}_{\mathrm{t}^{\prime}}\left(\theta_{\mathrm{i}}\left(\mathrm{t}^{\prime}\right)\right)$ has three contact pairs, namely, a triple-contact placement with $<\mathrm{e}, \mathrm{v}>$ between $\mathrm{A}$ and $\mathrm{P}, \omega_{\mathrm{i}}$, and $\omega_{\mathrm{i}-1}\left(\right.$ or $\left.\omega_{\mathrm{i}+1}\right)$. We are therefore led to the following lemma.

Lemma 3: A point on edge e of $\mathrm{A}$ is a Type $\mathrm{V}$ critical point 
with respect to a vertex $v$ of polygon $P \in \Xi$ if and only if there is a triple-contact placement of A with one of contact pairs being $<\mathrm{e}, \mathrm{v}>$.

\section{COMPUTING TRIPE-CONTACT PLACEMENTS}

The result of the analyses of the previous section calls for the identification of all the triple-contact placements of A. Consider a triple-contact placement of A with three contact pairs $\omega_{1}, \omega_{2}, \omega_{3}$, each with one of distinct polygons $\mathrm{P}_{1}, \mathrm{P}_{2}$, and $\mathrm{P}_{3}$ respectively. To maintain the first contact $\omega_{1}$ between $\mathrm{A}$ and $\mathrm{P}_{1}$ results in a ruled surface in the configuration space. Parameterize this ruled surface by $(t, \theta)$. To simultaneously maintain the second contact $\omega_{2}$ between $\mathrm{A}$ and $\mathrm{P}_{2}$ introduces a constraint relation between $t$ and $\theta$; this forms a critical curve $\gamma_{1}$ in the parameter plane $(\mathrm{t}, \theta)$. In the same way, the contact between $A$ and $P_{3}$ introduces another critical curve $\gamma_{2}$ in $(t, \theta)$. The image of the intersection point of these two critical curves on the ruled surface is then a triple-contact point in configuration space (cf. Figure 16). The parametric forms of three different types of critical curves are presented in Appendix. A generic procedure for computing all the triple-contact placements is given below.

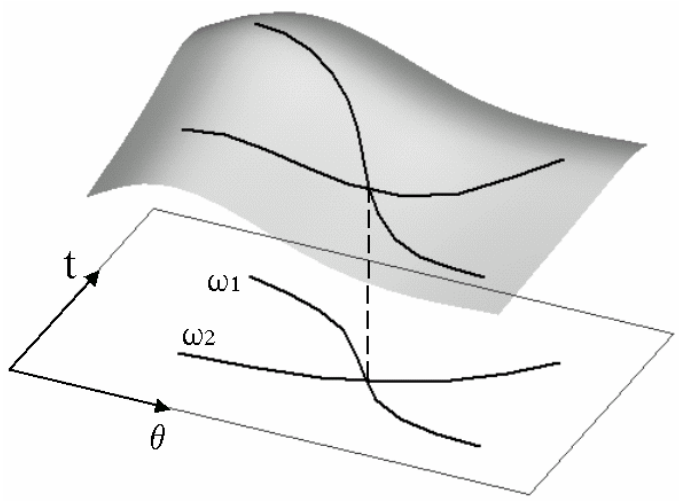

Fig. 16. The computation of a triple-contact placement

Algorithm: Triple_Contact_Placements (A, $\Xi)$

$/ *$ find all the triple-contact placements of A with respect to $\Xi * /$ begin

Step 1. $\mathrm{S} \leftarrow \varnothing$;

Step 2. for every distinct three polygons $\mathrm{P}_{\mathrm{i}}, \mathrm{P}_{\mathrm{j}}$, and $\mathrm{P}_{\mathrm{k}}$ in $\Xi$ do begin

Step 2.1. for every contact pair $\omega_{1}, \omega_{2}$, and $\omega_{3}$ (between $A$ and $\mathrm{P}_{\mathrm{i}}, \mathrm{P}_{\mathrm{j}}$, and $\mathrm{P}_{\mathrm{k}}$ respectively) do begin

Step 2.1a. $\quad \gamma_{1} \leftarrow$ the critical curve of $\omega_{1}, \omega_{2}$

Step 2.1b. $\quad \gamma_{2} \leftarrow$ the critical curve of $\omega_{1}, \omega_{3}$

Step 2.1c. $\quad \tau \leftarrow \gamma_{1} \cap \gamma_{2}$ end

if $\tau$ exists then $\mathrm{S} \leftarrow \mathrm{S} \cup\{\tau\}$

end

end
To analyze the running time of the above algorithm, let $n_{i}$ be the number of edges on polygon $P_{i}, i=1,2, \ldots, n$. For every vertex and edge on $\mathrm{A}$ and the three polygons $\mathrm{P}_{\mathrm{i}}, \mathrm{P}_{\mathrm{j}}$, and $\mathrm{P}_{\mathrm{k}}$, we need to check if the three corresponding contact pairs $\omega_{1}, \omega_{2}$, and $\omega_{3}$ constitute a triple-contact placement. Thus, the total number of iterations at Step 2.1 is $8 b^{3} n_{i} n_{j} n_{k}$, where $b$ is the number of edges on A. Since all the three steps Step 2.1a through Step 2.1c take a constant time (cf. Appendix), the entire loop at Step 2.1 requires $O\left(b^{3} n_{i} n_{j} n_{k}\right)$ to compute. Therefore the total running time of algorithm Triple_Contact_Placements is $\mathrm{O}\left(\mathrm{b}^{3} \sum_{i, j, k=1}^{n} i \neq j \neq k=n_{i} n_{j} n_{k}\right)$, which can be easily shown to be less than $\mathrm{O}\left(\mathrm{b}^{3} \mathrm{E}^{3}\right)$ where $\mathrm{E}=\sum_{i=1}^{n} n_{i}$ is the number of edges in $\Xi$.

\section{AlgORITHM DETAILS AND COMPLEXITY ANALYSIS}

With the types of critical points classified and their geometric characterizations established, this section presents the final algorithm that finds a representative set of $\pi(\mathrm{A}, \Xi)$, as well as its complexity analysis. Let $\left\{a_{1}, a_{2}, \ldots, a_{b}\right\}$ be the $b$ vertices on $A$ and $E$ be the total number of edges on the $n$ polygons in $\Xi=\left\{\mathrm{P}_{1}, \mathrm{P}_{2}, \ldots, \mathrm{P}_{\mathrm{n}}\right\}$. The core of the algorithm consists of two routines, Representative_VE and Representative_EV, that compute $\Sigma^{*}(\omega)$ for a contact pair $\omega$ of V-E and E-V type, respectively. The outline of the algorithm is given below.

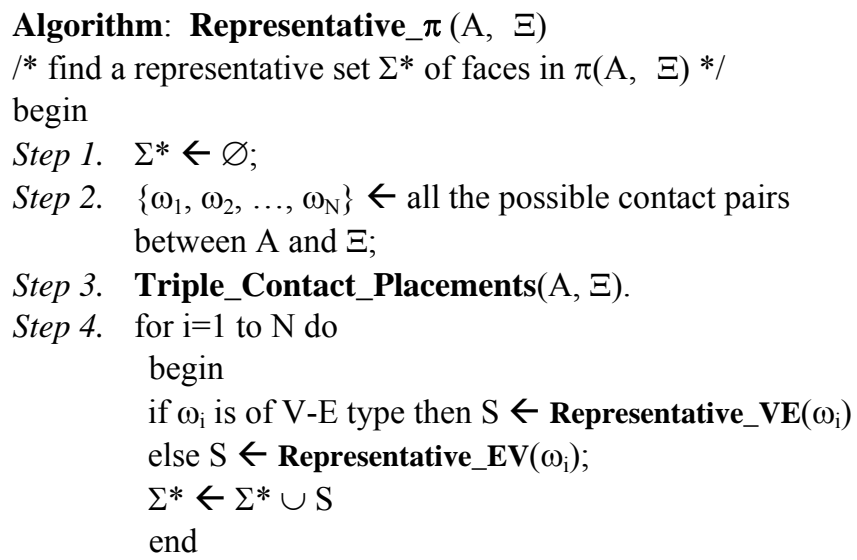

Step 5. Output $\Sigma^{*}$. end

There are exactly $2 b E$ possible contact pairs, each between one of the $b$ vertices (resp. edges) of $A$ and one of $E$ edges (resp. vertices) in $\Xi$. The two routines Representative_VE $\left(\omega_{\mathrm{i}}\right)$ and Representative_EV $\left(\omega_{\mathrm{i}}\right)$ called in Step 4 are responsible for finding a representative set $\mathrm{S}$ of $\pi\left(\omega_{\mathrm{i}}\right)$ which is then appended to the final set $\Sigma^{*}$.

By perturbing the set $\Sigma^{*}$ in linear time, we obtain a finite representative set $\Sigma$. This set is a superset of a kernel representation: each kernel in configuration space is represented by a subset of elements in $\Sigma$, which serve as the sample points of that kernel. This sampling is sufficient for 
discretization since it results from perturbing every vertex of that kernel. If a concise representation of kernels is needed, we index the polygons in $\Xi$ and then sort $\Sigma$ keyed on the index values. For all the CC points in $\Sigma$ having an identical key value, we keep only one copy in $\Sigma$ by deleting others. By using a quick sort algorithm [5], this clean up process takes expected time $\Theta($ log $)$, where $p$ is the number of elements in the representative set.

\section{A. Algorithm Representative_VE}

Let $\mathrm{v}$ be a vertex on $A$ and $\mathrm{e}$ be an edge in $\Xi$ and note that all the Type III critical points of contact pair $\langle\mathrm{v}$,e $>$ on edge e have already been identified by algorithm Triple_Contact_ Placements(A, $\Xi)$.

\section{Algorithm: Representative_VE $(<\mathrm{v}, \mathrm{e}>)$}

begin

Step 1. $\mathrm{S} \leftarrow \varnothing$;

Step 2. Calculate Type I critical points on e;

Step 3. Calculate Type II critical points on e;

Step 4. Sort all the critical points (Types I, II, III) on e to form the congruent partitioning of e;

Step 5. for each congruent interval $\left(t_{i}, t_{i+1}\right)$ do begin end computing the eigen set $\mathrm{Ei}_{<\mathrm{v}, \mathrm{e}}\left(\mathrm{t}_{\mathrm{i}}, \mathrm{t}_{\mathrm{i}+1}\right) \rightarrow \mathrm{S}$

Step 6. Output S.

end

\section{1) Calculating Critical Points}

Let $\mathrm{m}_{\mathrm{I}}, \mathrm{m}_{\mathrm{II}}$ and $\mathrm{m}_{\text {III }}$ be the numbers of Types I, II and III critical points of contact pair $\langle\mathrm{v}, \mathrm{e}\rangle$, respectively. At steps 2 and 3, only the Type I and II need to be checked. For Type I, Figure 6 clearly illustrates how a vertex or edge in $\Xi$ should be examined to see if it will contribute a Type I critical point on e. As there are only E vertices and edges to check, the time required for checking Type I points on e is thus $\mathrm{O}(\mathrm{bE})$. Since owing to the convexity a polygon in $\Xi$ can contribute at most two Type I critical points to a pair $<\mathrm{v}, \mathrm{e}>$, the number $\mathrm{m}_{\mathrm{I}}$ is at most 2(n-1). For Type II, referring to Figure 7, we need first to build offset $C_{L}\left(P_{i}\right)$ for all the polygons $P_{i}$ in $\Xi$, intersect edge e with these offsets next, and then identify those intersection points where the contact condition as stipulated in Figure 7 is satisfied. It takes linear time $\mathrm{O}(\mathrm{E})$ to build these offsets and obviously the same time to intersect them with edge e. It is readily seen that $\mathrm{m}_{\mathrm{II}}$ is also at most $2(\mathrm{n}-1)$ (edge e intersects a $\mathrm{C}_{\mathrm{L}}\left(\mathrm{P}_{\mathrm{i}}\right)$ at most twice). Obviously, $\mathrm{O}(\mathrm{E}+\mathrm{b})$ time is sufficient for identifying all the $\mathrm{m}_{\text {II }}$ Type II critical points of $\langle\mathrm{v}, \mathrm{e}\rangle$. The following lemma is in order.

Lemma 4: Assuming all the triple-contact placements have already been identified, it takes $\mathrm{O}(\mathrm{bE})$ time to find all the critical points of a $\mathrm{V}$-E type contact pair $\langle\mathrm{v}$,e $>$ on edge e, and the total number of critical points of $\langle\mathrm{v}, \mathrm{e}\rangle$ on $\mathrm{e}$ is less than $4 n+\mathrm{m}_{\text {III. }}$.

By applying Lemma 4 to all the possible bE V-E type contact pairs and noticing the amortization of $\mathrm{m}_{\mathrm{III}}$ on all the edges in $\Xi$, with the result of Section 4, we have the following result on the total number of critical points of all the possible V-E type contact pairs on all the edges in $\Xi$.

Theorem 1: Let $\mathrm{M}_{\mathrm{I}}, \mathrm{M}_{\mathrm{II}}$, and $\mathrm{M}_{\mathrm{III}}$ respectively be the number of Type I, Type II, and Type III critical points of all the possible $\mathrm{V}$-E type contact pairs between $\mathrm{A}$ and $\Xi$. Then they are bounded by $\mathrm{O}(\mathrm{bnE}), \mathrm{O}(\mathrm{bnE})$, and $\mathrm{O}\left(\mathrm{b}^{3} \mathrm{E}^{3}\right)$ respectively and can be computed in time $\mathrm{O}\left(\mathrm{b}^{2} \mathrm{E}^{2}\right), \mathrm{O}\left(\mathrm{b} \mathrm{E}^{2}+\mathrm{b}^{2} \mathrm{E}\right)$ and $\mathrm{O}\left(\mathrm{b}^{3} \mathrm{E}^{3}\right)$, respectively.

It must be emphasized that a critical point is meaningful only with respect to a particular contact pair $\langle\mathrm{v}, \mathrm{e}\rangle$. After all the critical points of $\langle\mathrm{v}, \mathrm{e}>$ are identified, they are sorted so to form congruent intervals on edge e. The next task is then to find an eigen set for each of these intervals.

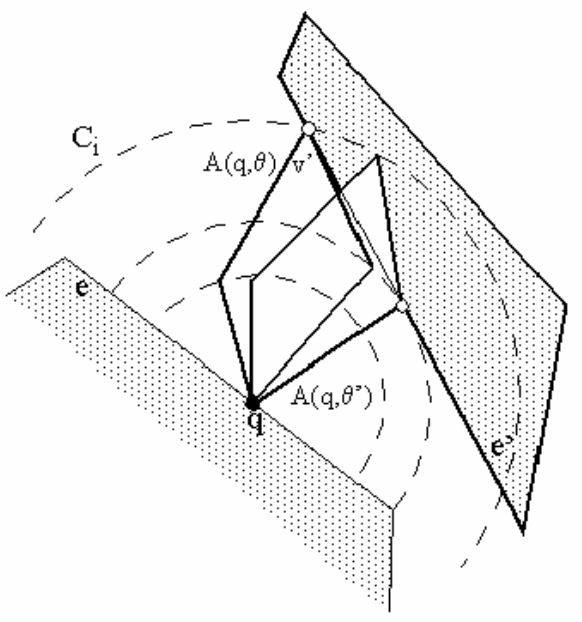

(a)

(b)

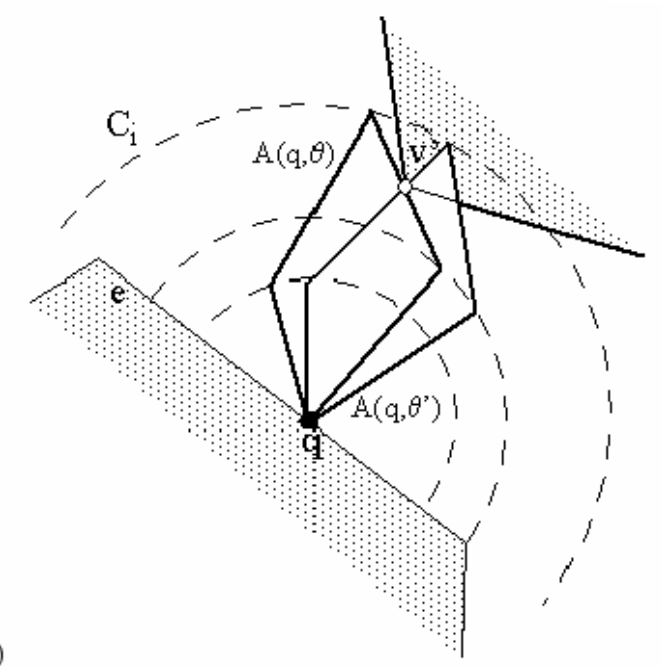

Fig. 17. Eigen set calculation

\section{2) Calculating Eigen Sets}

To find an eigen set of a congruent interval I on edge e, we choose an arbitrary point $\mathrm{q}$ from the interior of $\mathrm{I}$ and then construct the angular partitioning at q. Referring to Figure 17, to build the angular partitioning at $\mathrm{q}$, a set of co-centric semi-circles $\mathrm{C}_{\mathrm{i}}$ are defined that center at $\mathrm{q}$ and have radii equal to the distances between vertex $\mathrm{v}$ and other vertices on $\mathrm{A}$ 
respectively. For each edge e' in $\Xi$ (exclusive of those on the same polygon with e), if it intersects a $C_{i}$, the intersection point determines a unique instance $\mathrm{A}(\mathrm{q}, \theta)$ (assuming $\mathrm{v}$ is the reference point of $A$ ) whose vertex $v$ ' (which defines $C_{i}$ ) coincides with the intersection point. Number $\theta$ is in the angular partitioning of $q$ if and only if edge e' touches but not intersects $\mathrm{A}(\mathrm{q}, \theta)$, e.g., in Figure $17(\mathrm{a}) \theta$ is but $\theta^{\prime}$ is not in the angular partitioning of q. The examination on a vertex v' in $\Xi$ is similar to that of edges, except this time $\mathrm{v}^{\prime}$ is checked against a ring between some neighboring $\mathrm{C}_{i}$ and $\mathrm{C}_{\mathrm{i}+1}$, as manifested by Figure 17(b). Observing that for any polygon $P_{i}$ in $\Xi$ there exist at most two instances $A(q, \theta)$ and $A\left(q, \theta^{\prime}\right)$ to contact $P_{i}$ (owing to the convexity of both $\mathrm{P}_{\mathrm{i}}$ and $\left.\mathrm{A}\right)$, the following lemma is in order.

Lemma 5: It takes $\mathrm{O}(\mathrm{b} \log \mathrm{b}+\mathrm{bE}+\mathrm{n} \log \mathrm{n})$ time to compute an eigen set of a point q on an edge e in $\Xi$ with respect to a vertex $\mathrm{v}$ of $\mathrm{A}$, and the number of points in the eigen set is less than $2 \mathrm{n}$.

In deriving the above lemma, the first term blogb is due to the need to build the $b-1$ rings out of the $b-1$ semi-circles $C_{i}$. The term $\mathrm{bE}$ is obviously required for checking every vertex and edge in $\Xi$ against the circles $C_{i}$ and verifying the correct contact. The last term nlogn owes to the fact that the angles in the angular partitioning have to be sorted so that the eigen set can be obtained.

Given a contact pair $\left\langle\mathrm{v}\right.$, e $>$, the numbers $\mathrm{m}_{\mathrm{I}}, \mathrm{m}_{\mathrm{II}}$ and $\mathrm{m}_{\text {III }}$ of type I, II and III critical points on e are bounded by $\mathrm{O}(\mathrm{n}), \mathrm{O}(\mathrm{n})$ and $\mathrm{O}\left(\mathrm{b}^{2} \mathrm{E}^{2}\right)$, respectively. Then sorting the critical points with respect to all the possible $\mathrm{V}$-E type contact pairs takes time $\mathrm{O}\left(\mathrm{b}^{3} \mathrm{E}^{3} \log (\mathrm{bE})\right)$. Together, the following result is presented.

Theorem 2: There is a representative set $\Sigma^{*}$ of $\pi(\mathrm{A}, \Xi)$ in which the number of V-E contact type points is bounded by $\mathrm{O}(\mathrm{nM})$ and they can be found in $\mathrm{O}\left(\mathrm{b}^{3} \mathrm{E}^{3} \log (\mathrm{bE})+\mathrm{M}(\mathrm{b} \log \mathrm{b}+\mathrm{bE}+\right.$ nlogn)) time, where $\mathrm{M}$ is the total number of type I, II, and III critical points with respect to all the contact pairs $<\mathrm{v}$, e $>$ between $\mathrm{A}$ and $\Xi$, which is bounded by $\mathrm{O}\left(\mathrm{b}^{3} \mathrm{E}^{3}\right)$.

\section{B. Algorithm Representative_EV}

Recall that Type IVa critical points are identical to Type I critical points and Type $\mathrm{V}$ critical points belong to triple-contact placements of A. Thus, Type IVa and V critical points can be identified by performing algorithm Representative_VE and Triple_Contact_Placements(A, $\Xi$ ), respectively. Let e be an edge on $\mathrm{A}$ and $\mathrm{v}$ be a vertex in $\Xi$.

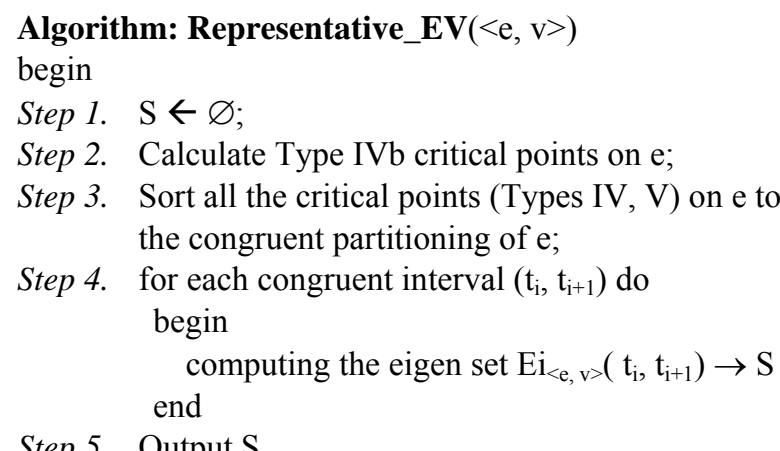

Step 5. Output S. end

The algorithm Representative_EV is similar to that of Representative_VE; the only difference lies in critical points identification. To identify Type IVb critical points, the following lemma is needed.

Lemma 6: Given a contact pair $<\mathrm{e}, \mathrm{v}>, \mathrm{e} \in \mathrm{A}, \mathrm{v} \in \mathrm{P} \in \Xi$, and a covering radius $\mathrm{R}$, the number of Type $\mathrm{IVb}$ critical points associated with $\langle\mathrm{e}, \mathrm{v}\rangle$ is at most two and can be identified in $\mathrm{O}(\mathrm{b})$ time. Thus the total number of Type IVb critical points between $\mathrm{A}$ and $\Xi$ is bounded by $\mathrm{O}(\mathrm{bnE})$ and can be identified in $\mathrm{O}\left(\mathrm{b}^{2} \mathrm{nE}+\mathrm{E}^{2}\right)$ time.

Proof. Given a contact pair $<\mathrm{e}, \mathrm{v}>$ and a covering radius $\mathrm{R}$, suppose there are $\mathrm{m}$ Type IVb critical points on e contributed by $m$ vertices in $A$. Let $\Lambda$ be the intersection of the $m$ circular discs of radius $\mathrm{R}$, with centers at the $\mathrm{m}$ critical points respectively. By definition of a Type IVb point, $\Lambda$ must enclose polygon A. In addition, each of the $m$ contributing vertices lies on a distinct arc on the boundary of $\Lambda$ (cf. Figure 15(b)). Since all the $\mathrm{m}$ critical points lie on a same line, the boundary of the intersection $\Lambda$ can only consist of two distinct arcs (see Figure 18); consequently, m can not exceed 2 .

We have to go through each of the b vertices in A to check whether it contributes Type IVb critical point; therefore, it takes $\mathrm{O}(\mathrm{b})$ time. Due to the convexity, every polygon in $\Xi$ can offer at most one possible covering radius $\mathrm{R}$ for a given vertex in $\Xi$. Identifying all the covering radii between $A$ and $\Xi$ takes time $\mathrm{O}\left(\mathrm{E}^{2}\right)$. Thus it is readily verified that the total number of Type IVb critical points between $A$ and $\Xi$ is bounded by $\mathrm{O}(\mathrm{bnE})$ and can be identified in $\mathrm{O}\left(\mathrm{b}^{2} \mathrm{nE}+\mathrm{E}^{2}\right)$ time.

Q.E.D.

Sorting the critical points is the same as that of V-E type. Given a point $\mathrm{q}$ on e of $\mathrm{A}$, to calculate its associated eigen set is similar to that of V-E type by just replacing the semi-circles with full circles.

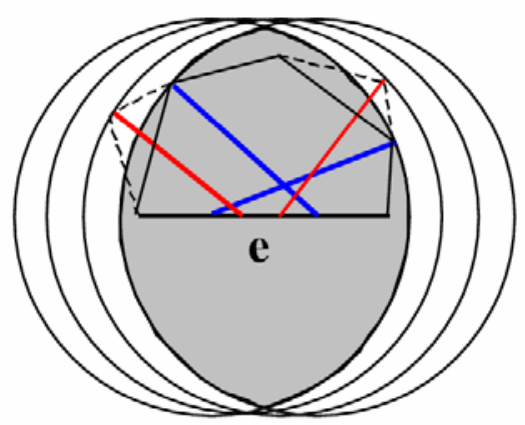

Fig. 18. Proof of Lemma 6

\section{Algorithm Complexity}

The complexity of the proposed algorithm clearly depends on the number of critical points, and thus, the algorithm is output-sensitive:

Theorem 3: Let $\mathrm{M}=\mathrm{M}_{\mathrm{tcp}}+\mathrm{M}_{\text {others }}$ be the total number of critical points of all the types between $A$ and $\Xi$, where $M_{\text {tcp }}$ is 
the number of all the triple-contact placements of A which is bounded by $\mathrm{O}\left(\mathrm{b}^{3} \mathrm{E}^{3}\right)$ and $\mathrm{M}_{\text {others }}$ is the number of other critical points which is bounded by $\mathrm{O}(\mathrm{bnE})$. The elements in the representative set $\Sigma^{*}$ of $\pi(\mathrm{A}, \Xi)$ is then bounded by $\mathrm{O}(\mathrm{nM})$ and can be found in $\mathrm{O}\left(\mathrm{b}^{3} \mathrm{E}^{3}+\mathrm{M} \log \mathrm{M}+\mathrm{M}(\mathrm{b} \log \mathrm{b}+\mathrm{bE}+\mathrm{n} \log \mathrm{n})\right)$.

The complexity of the output-sensitive algorithm depends on the arrangement of the polygonal environment $\Xi$. It is inviting to ask whether the upper bound $\mathrm{O}\left(\mathrm{b}^{3} \mathrm{E}^{3}\right)$ for $\mathrm{M}_{\mathrm{tcp}}$ is tight or not. The answer to this is better exploited from the point of view of the configuration space. In the configuration space, the number of all the boundary surfaces of all the C-primitives is clearly $\mathrm{O}(\mathrm{bE})$. Since each boundary surface is a ruled surface with (small) constant maximum degree and, in the most general case, the polygons in $\Xi$ are allowed to overlap, $\mathrm{O}\left(\mathrm{b}^{3} \mathrm{E}^{3}\right)$ is a worse-case tight bound on the number of all the vertices in any three-dimensional arrangement of $\mathrm{O}(\mathrm{bE})$ algebraic surface patches of constant maximum degree. Prior to this general upper bound, we note that better bounds have been achieved for certain special applications: Leven and Sharir [16] showed that, if all the polygons in $\Xi$ are convex and disjoint, the total number of triple-contact placements of $\mathrm{A}$ is bounded by $\mathrm{O}\left(\mathrm{bE} \lambda_{6}(\mathrm{bE})\right)$, where $\lambda_{\mathrm{s}}(\mathrm{x})$ is the maximum length of $(\mathrm{x}, \mathrm{s})$-Davenport-Schinzel sequences [22], and all these critical points can be computed in time $\mathrm{O}\left(\mathrm{bE} \lambda_{6}(\mathrm{bE}) \log (\mathrm{bE})\right)$ [13].

It is also interesting to exploit what is the average-case running time of the proposed algorithm in the most general case. A rigorous analysis on the average case depends on both the algorithm implementation and a large number of practical tests constituting an "average" input for a particular application, which is nevertheless beyond the scope of this paper.

\section{Algorithm Implementation}

To demonstrate its feasibility, the proposed algorithm is implemented on the Visual C++ platform. Figure 19 illustrates the experimental result with both a vertex-edge contact type and an edge-vertex contact type. In the first example (cf. Figure $19(\mathrm{a})), \Xi=\left\{\mathrm{P}_{1}, \mathrm{P}_{2}, \mathrm{P}_{3}, \mathrm{P}_{4}\right\}$ and all the critical points on an edge $\mathrm{e} \in \mathrm{P}_{1}$ with respect to a particular contact pair $\langle\mathrm{v}, \mathrm{e}>, \mathrm{v} \in \mathrm{A}$, are presented. The critical points of different types are displayed with different symbols. The angular partitioning of $\mathrm{q} \in\left(\mathrm{t}_{11}, \mathrm{t}_{12}\right)$ is also displayed with dashed lines. In the second example (cf. Figure 19(b)), $\Xi=\left\{\mathrm{P}_{1}, \ldots, \mathrm{P}_{8}\right\}$ and all the critical points on an edge $e \in A$ with respect to a vertex $v \in P_{1}$ are displayed; the contact order of point $\mathrm{e}(\mathrm{t}) \in\left(\mathrm{t}_{18}, \mathrm{t}_{19}\right)$ is also displayed with dashed lines.

The simple implementation described here is however not sufficient to test the efficiency and robustness of the algorithm. The details of the issues on reliability and numerical accuracy with floating-point arithmetic, as well as degenerate cases handling, are out of the scope of this paper and will be presented in a follow-up paper with a large set of practical experiments.

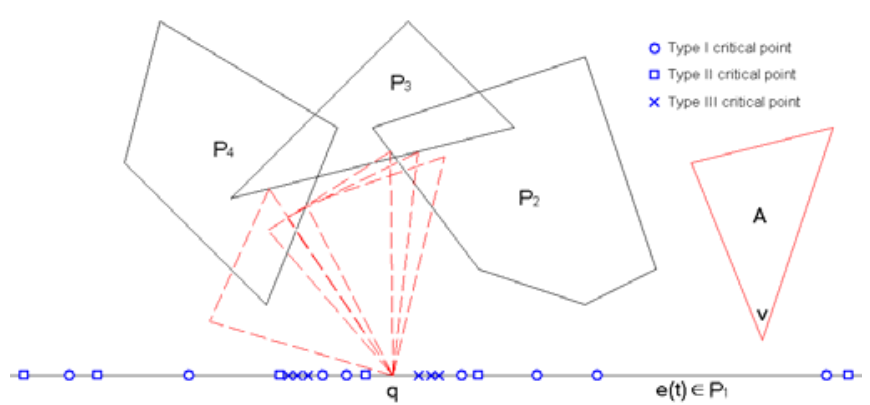

(a) The experimental result with a vertex-edge contact type

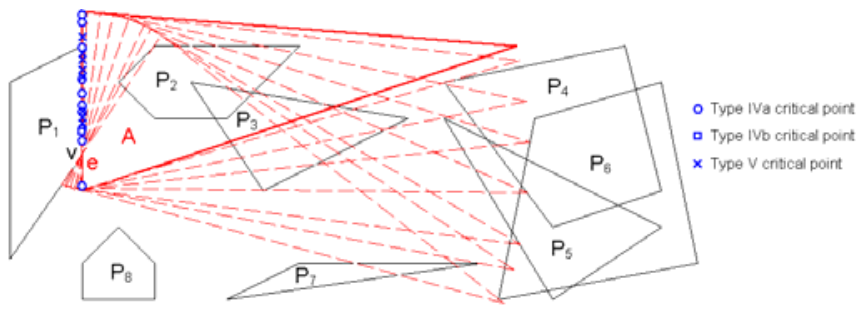

(b) The experimental result with an edge-vertex contact type

Fig. 19. Algorithm implementation

\section{APPLICATION SCENARIO}

Consider the following geometric optimization problem. In a constrained planar region $\mathrm{D}$, given a set $\Xi$ of planar convex polygons among which some are "hard" (its meaning given below), and given a convex polygon A:

1) Can A be placed within D without touching any polygons in $\Xi$ ?

2) If not, what is the minimum number of polygons in $\Xi$ that need to be removed such that A can be placed within D?

3) If the "hard" polygons in $\Xi$ cannot be removed, what is the minimum number of polygons in $\Xi$ that need to be removed such that A can be placed within D?

An important application of the answers to these questions is in the landscape development: if we want to build a rathaus in the downtown which is full of old commercial and residential buildings, can we find such a place for rathaus or what is the minimum number of buildings that need to be demolished for such a placement? In the downtown, the nearby highway, parks, museums, or historical sites are hard constraints that should be preserved. The convex hull of the $2 \mathrm{D}$ projection of the rathaus corresponds to A (cf. Figure 20(a)), the downtown area is D and the downtown buildings form $\Xi$ including hard ones (the colored ones in Figure 20(b)). Note that the 2D projections of buildings can overlap each other.

The first question posed above might be answered by existing algorithms, but not the second nor the third. To place a polygon $\mathrm{A}$ within $\mathrm{D}$, we decompose the outside region that complements region $\mathrm{D}$ in the plane using a set of unbounded convex polygons which are treated as hard constraints (cf. Figure 21) and included in $\Xi$. By applying the proposed algorithm to $A$ and $\Xi$, we obtain an intersection representative set $\Sigma$ from which we first delete all the placements whose 
intersection polygon list contains hard polygons. The remaining placements in $\Sigma$ are then sorted keyed on the intersection polygon number in ascent order and the element on the top of the list is the answer. In the case shown in Figure 20, we have three candidate placements of $\mathrm{A}$ which require removing only two "soft" polygons (cf. Figure 21). The final, exact location of A can be obtained by considering further optimization criteria, e.g., minimizing the overlap area [21].

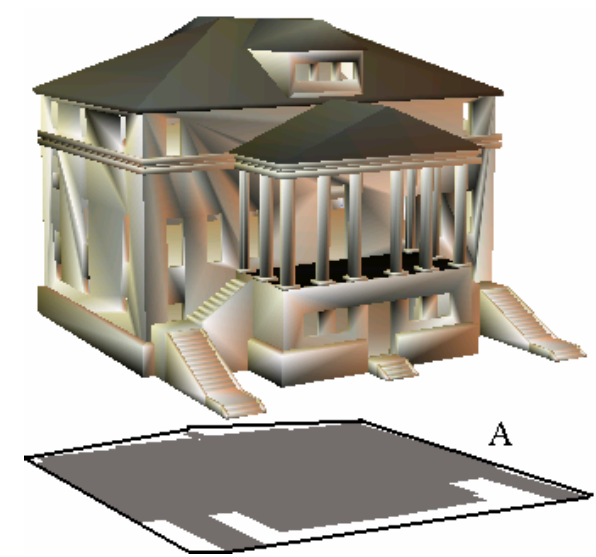

(a) A rathaus and its $2 \mathrm{D}$ projection

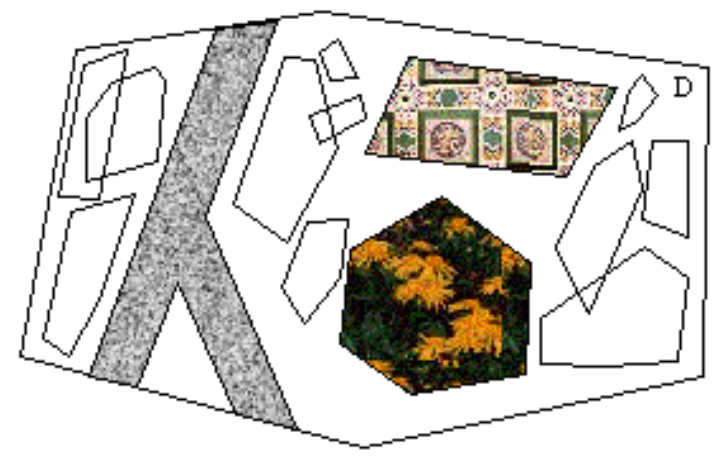

(b) The zoomed downtown map

Fig. 20. The landscape development problem

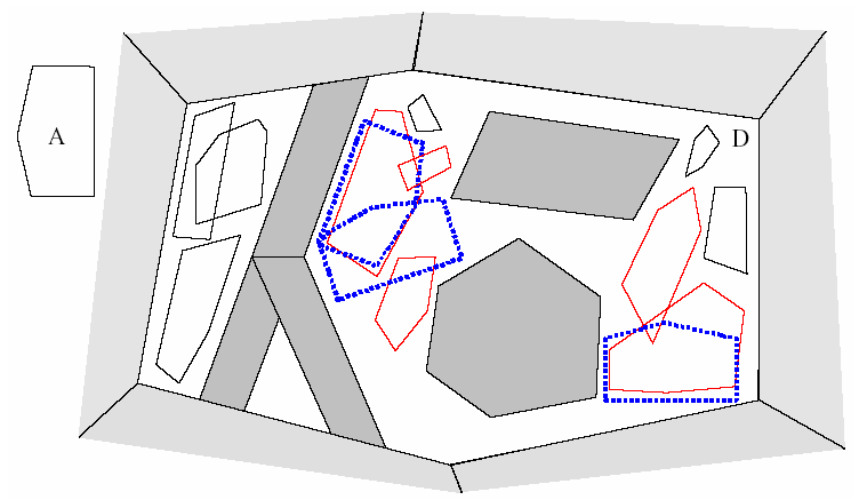

Fig. 21. A solution to the landscape development problem in Fig. 20

\section{CONCLUSION}

In this paper we propose a geometric method to determine a finite and discrete representation of all the possible intersection relations between a movable convex object and a set of planar convex polygons. The main contribution is that, by formulating the intersection relations with the topological structure of kernels decomposition in configuration space, we identify all the possible intersection relations completely by their geometric realizations in object space; therefore, complex algebraic formulation and tedious intersection testing and trimming are avoided. A construction algorithm is proposed with time and space efficiency and an interesting example in landscape development is presented to demonstrate the rich applications of the formulated problem and the proposed solution.

In terms of further improvement of the proposed algorithm and possible future research, three points are noted below.

1) In principle, the basic ideas of the paper are applicable to a curved environment, i.e., the objects in $\Xi$ are simple and compact figures bounded by convex simple curves such as Bezier curves. In [27] a detailed algebraic description on the contact between a convex polygon and a convex curved figure is given. Rigorous analyses as well as efficient algorithms are however needed to formulate and compute all the types of critical points. Especially, how to identify a triple-contact placement between three curved edges poses to be a challenging task.

2) The complexity analysis given in the paper on the proposed algorithm is rather coarse and it should be inquired if better bounds on the running time can be achieved. Moreover, from practical point of view, it is also imperative that the proposed algorithm be completely implemented and applied to a large set of test data, so to verify its robustness in dealing with geometric degeneracy as well as to study the average running time. A follow-up paper concentrating on both the theoretical algorithmic analysis and the experiments is current in progress and hopefully it can address the above two issues.

3) One interesting extension is to transform the domain from the plane to the sphere - finding all the intersection relations between a movable spherical polygon and a set of fixed spherical polygons (all on the unit sphere). By consolidating the spherical polygons with visibility maps, one important application of this spherical geometry problem is in 5-axis $\mathrm{NC}$ machining [26]: finding a set-up for a 5-axis NC machine so that the maximal area of a given free-form part can be machined. This optimization problem can be formulated as finding a placement of a given spherical polygon that intersects a maximal number of a set of fixed spherical polygons.

\section{APPENDIX}

In this appendix, we establish the parametric formulation of critical curves. As illustrated in Figure A-1, given a local 2D coordinate frame $\left\{\mathbf{e}_{\mathrm{x}}, \mathbf{e}_{\mathrm{y}}\right\}$ and a global $2 \mathrm{D}$ coordinate frame $\left\{\mathbf{g}_{\mathrm{x}}\right.$, $\left.\mathbf{g}_{\mathrm{y}}\right\}$, the transformation rule $\mathrm{H}$ mapping the point $\left(\mathrm{x}_{\mathrm{e}}, \mathrm{y}_{\mathrm{e}}\right)$ in $\left\{\mathbf{e}_{\mathrm{x}}\right.$, $\left.\mathbf{e}_{\mathrm{y}}\right\}$ to the point $\left(\mathrm{x}_{\mathrm{g}}, \mathrm{y}_{\mathrm{g}}\right)$ in $\left\{\mathbf{g}_{\mathrm{x}}, \mathbf{g}_{\mathrm{y}}\right\}$ is 


$$
\left(\begin{array}{l}
x_{g} \\
y_{g}
\end{array}\right)=\left(\begin{array}{cc}
\cos \theta & -\sin \theta \\
\sin \theta & \cos \theta
\end{array}\right)\left(\begin{array}{l}
x_{e} \\
y_{e}
\end{array}\right)+\left(\begin{array}{l}
o_{x} \\
o_{y}
\end{array}\right)
$$

or in matrix form

$$
\mathbf{p}_{g}=H\left(\mathbf{p}_{e}\right)=\mathbf{R} \mathbf{p}_{e}+\mathbf{T}
$$

where $\mathbf{R}$ and $\mathbf{T}$ stand for the rotation matrix and translation vector, respectively. A vector $\mathbf{v}_{\mathrm{e}}=\mathbf{a}_{\mathrm{e}}-\mathbf{b}_{\mathrm{e}}$ in $\left\{\mathbf{e}_{\mathrm{x}}, \mathbf{e}_{\mathrm{y}}\right\}$ is then mapped to a vector $\mathbf{v}_{\mathrm{g}}$ in $\left\{\mathbf{g}_{\mathrm{x}}, \mathbf{g}_{\mathrm{y}}\right\}$ by

$$
\mathbf{v}_{g}=\mathbf{a}_{g}-\mathbf{b}_{g}=H\left(\mathbf{a}_{e}\right)-H\left(\mathbf{b}_{e}\right)=\mathbf{R}\left(\mathbf{a}_{e}-\mathbf{b}_{e}\right)=\mathbf{R} \mathbf{v}_{e}
$$

Given the point and vector transformation rules, we analyze the following three types of critical curves.

\section{A. Critical Curve $\alpha$ with V-E and V-E Contact Pairs}

In this case, the critical curve is a special version of sliding curve [25], which results from two contact pairs $<\mathrm{a}_{\mathrm{i}}, \mathrm{E}_{\mathrm{m}}>$ and $<a_{j}, E_{n}>$, where $a_{i}$ and $a_{j}$ are two distinct vertices of $A$, and $E_{m}$ and $E_{n}$ are two distinct edges of $P_{m} \in \Xi$ and $P_{n} \in \Xi$, respectively, $\mathrm{P}_{\mathrm{m}} \neq \mathrm{P}_{\mathrm{n}}$.

As illustrated in Figure A-2, we assign the local coordinate frame $\left\{\mathbf{o}_{\mathrm{e}}, \mathbf{e}_{\mathrm{x}}, \mathbf{e}_{\mathrm{y}}\right\}$ by $\mathbf{o}_{\mathrm{e}}=\mathbf{a}_{\mathrm{i}}, \mathbf{e}_{\mathrm{x}}=\mathbf{e}_{\mathrm{i}}, \mathbf{e}_{\mathrm{y}} \perp \mathbf{e}_{\mathrm{i}}$, where $\mathbf{e}_{\mathrm{i}}$ is the edge of $\mathrm{A}$ incident to $\mathbf{a}_{\mathrm{i}}, \mathbf{b}_{\mathrm{m}}$ is a vertex incident to $\mathrm{E}_{\mathrm{m}}$ and $\mathbf{b}_{\mathrm{n}}$ is a vertex incident to $E_{n}$. The normalized direction along $E_{m}$ and $E_{n}$ are denoted by $\mathbf{E}_{\mathrm{m}}$ and $\mathbf{E}_{\mathrm{n}}$, respectively. Denoting the position of point $\mathbf{a}_{\mathrm{j}}$ in $\left\{\mathbf{o}_{\mathrm{e}}, \mathbf{e}_{\mathrm{x}}, \mathbf{e}_{\mathrm{y}}\right\}$ as $\mathbf{a}_{\mathrm{ji}}$ and parameterizing the placement of A by $(t, \theta)$ as shown in Figure A-2, we have

$$
\begin{gathered}
\mathbf{a}_{i}=\mathbf{b}_{m}+t \mathbf{E}_{m} \\
\mathbf{a}_{j}=H\left(\mathbf{a}_{j i}\right)=\mathbf{R}(\theta) \mathbf{a}_{j i}+\mathbf{a}_{i}=\mathbf{R}(\theta) \mathbf{a}_{j i}+\mathbf{b}_{m}+t \mathbf{E}_{m}
\end{gathered}
$$

with the constraint that $\mathbf{a}_{\mathbf{j}}$ lies on $\mathrm{E}_{\mathrm{n}}$,

$$
\begin{aligned}
& \mathbf{a}_{j}=\mathbf{b}_{n}+s \mathbf{E}_{n}=\mathbf{R}(\theta) \mathbf{a}_{j i}+\mathbf{b}_{m}+t \mathbf{E}_{m} \\
& \Rightarrow \mathbf{R}(\theta) \mathbf{a}_{j i}-s \mathbf{E}_{n}+t \mathbf{E}_{m}=\mathbf{b}_{n}-\mathbf{b}_{m} \Rightarrow \mathbf{R}(\theta) \mathbf{c}_{1}-s \mathbf{E}_{n}+t \mathbf{E}_{m}=\mathbf{c}_{2}
\end{aligned}
$$

where $\mathbf{c}_{1}, \mathbf{c}_{2}$ are constant column vectors. The above system has two equations (w.r.t. $\mathrm{x}$ and $\mathrm{y}$ directions) and three unknowns $\mathrm{s}$, $\theta$, t. In particular, the system is linear with respect to $\mathrm{s}$ and $\mathrm{t}$. Thus $t$ can be represented as a function with $\theta$ :

$$
\begin{aligned}
& \mathbf{R}(\theta) \mathbf{c}_{1}-s \mathbf{E}_{n}+t \mathbf{E}_{m}=\mathbf{c}_{2} \\
& \Rightarrow\left(\begin{array}{cc}
\cos \theta & -\sin \theta \\
\sin \theta & \cos \theta
\end{array}\right)\left(\begin{array}{l}
c_{11} \\
c_{12}
\end{array}\right)-s\left(\begin{array}{l}
E_{n 1} \\
E_{n 2}
\end{array}\right)+t\left(\begin{array}{l}
E_{m 1} \\
E_{m 2}
\end{array}\right)=\left(\begin{array}{l}
c_{21} \\
c_{22}
\end{array}\right) \\
& \Rightarrow\left\{\begin{array}{l}
t E_{m 1}-s E_{n 1}=c_{21}-\cos \theta \cdot c_{11}+\sin \theta \cdot c_{12} \\
t E_{m 2}-s E_{n 2}=c_{22}-\sin \theta \cdot c_{11}-\cos \theta \cdot c_{12}
\end{array} \Rightarrow\right.
\end{aligned}
$$

$\left(E_{m 1} E_{n 2}-E_{m 2} E_{n 1}\right) t=$

$c_{21} E_{n 2}-c_{22} E_{n 1}+\cos \theta \cdot\left(c_{12} E_{n 1}-c_{11} E_{n 2}\right)+\sin \theta \cdot\left(c_{12} E_{n 2}+c_{11} E_{n 1}\right)$

Assuming $E_{\mathrm{m} 1} E_{\mathrm{n} 2}-E_{\mathrm{m} 2} E_{\mathrm{n} 1} \neq 0$ (otherwise we get the trivial degenerate case of $\mathbf{E}_{\mathrm{m}} / / \mathbf{E}_{\mathrm{n}}$ ), we have

$$
\begin{aligned}
t & =a \cos \theta+b \sin \theta+d \\
& =\sqrt{a^{2}+b^{2}} \cos (\theta+\alpha)+d=c \cos (\theta+\alpha)+d
\end{aligned}
$$

where $\alpha, a, b, c$, and $d$ are constants determined by the geometry of $\mathrm{A}, \mathrm{P}_{\mathrm{m}}$, and $\mathrm{P}_{\mathrm{n}}$. The above equation introduces a critical curve in the parameter domain $(t, \theta)$ whose image on the parameterized ruled surface (a plane in this case) is the parameterized critical curve attributed to two $\mathrm{V}$-E type contact pairs.

\section{B. Critical Curve $\beta$ with $E-V$ and $E-V$ Contact Pairs}

In this case, the critical curve is a variation of isoptic curve [25], which results from two contact pairs $\left\langle e_{i}, b_{m}>\right.$ and $<e_{j}, b_{n}>$, where $e_{i}$ and $e_{j}$ are two distinct edges of $A$, and $b_{m}$ and $b_{n}$ are two distinct vertices of $P_{m} \in \Xi$ and $P_{n} \in \Xi$, respectively, $P_{m} \neq P_{n}$.

As illustrated in Figure A-3, we assign the local coordinate frame $\left\{\mathbf{o}_{\mathrm{e}}, \mathbf{e}_{\mathrm{x}}, \mathbf{e}_{\mathrm{y}}\right\}$ by $\mathbf{o}_{\mathrm{e}}=\mathbf{a}_{\mathrm{i}}, \mathbf{e}_{\mathrm{x}}=\mathbf{e}_{\mathrm{i}}, \mathbf{e}_{\mathrm{y}} \perp \mathbf{e}_{\mathrm{i}}$, where $\mathbf{a}_{\mathrm{i}}$ is the vertex of A incident to $\mathbf{e}_{i}, \mathbf{b}_{\mathrm{m}}$ is a vertex of $\mathrm{P}_{\mathrm{m}}$ and $\mathbf{b}_{\mathrm{n}}$ is a vertex of $\mathrm{P}_{\mathrm{n}}$. The normalized direction along $\mathrm{e}_{\mathrm{i}}$ and $\mathrm{e}_{\mathrm{j}}$ are denoted by $\mathbf{e}_{\mathrm{i}}$ and $\mathbf{e}_{\mathrm{j}}$, respectively. Denoting the presentation of point $\mathbf{a}_{j}$ and vector $\mathbf{e}_{j}$ in $\left\{\mathbf{o}_{\mathrm{e}}, \mathbf{e}_{\mathrm{x}}, \mathbf{e}_{\mathrm{y}}\right\}$ as $\mathbf{a}_{\mathrm{ji}}$ and $\mathbf{e}_{\mathrm{ji}}$, and parameterizing the placement of A by $(t, \theta)$, we have

$$
\begin{gathered}
\mathbf{a}_{i}=\mathbf{b}_{m}-\left(\begin{array}{c}
t \cos \theta \\
t \sin \theta
\end{array}\right) \Rightarrow \mathbf{a}_{i}=\mathbf{b}_{m}-T(t, \theta) \\
\mathbf{a}_{j}=H\left(\mathbf{a}_{j i}\right)=\mathbf{R}(\theta) \mathbf{a}_{j i}+\mathbf{a}_{i}=\mathbf{R}(\theta) \mathbf{a}_{j i}+\mathbf{b}_{m}-T(t, \theta) \\
\mathbf{e}_{j}=\mathbf{R}(\theta) \mathbf{e}_{j i} \\
\mathbf{b}_{n}=\mathbf{a}_{j}+s \mathbf{e}_{j}=\mathbf{R}(\theta) \mathbf{a}_{j i}+\mathbf{b}_{m}-T(t, \theta)+s \mathbf{R}(\theta) \mathbf{e}_{j i} \\
\Rightarrow \mathbf{R}(\theta) \mathbf{a}_{j i}+s \mathbf{R}(\theta) \mathbf{e}_{j i}-T(t, \theta)=\mathbf{b}_{n}-\mathbf{b}_{m} \\
\Rightarrow \mathbf{R}(\theta) \mathbf{c}_{1}+s \mathbf{R}(\theta) \mathbf{c}_{2}-T(t, \theta)=\mathbf{c}_{3}
\end{gathered}
$$

where $\mathbf{c}_{1}, \mathbf{c}_{2}, \mathbf{c}_{3}$ are constant column vectors. The above system has two equations (w.r.t. x, y directions) and three unknowns s, $\theta$, t. In particular, the system is linear with respect to $s$ and $t$. Thus we have:

$\mathbf{R}(\theta) \mathbf{c}_{1}+s \mathbf{R}(\theta) \mathbf{c}_{2}-T(t, \theta)=\mathbf{c}_{3}$

$\Rightarrow\left(\begin{array}{cc}\cos \theta & -\sin \theta \\ \sin \theta & \cos \theta\end{array}\right)\left(\begin{array}{l}c_{11} \\ c_{12}\end{array}\right)+s\left(\begin{array}{cc}\cos \theta & -\sin \theta \\ \sin \theta & \cos \theta\end{array}\right)\left(\begin{array}{l}c_{21} \\ c_{22}\end{array}\right)-t\left(\begin{array}{l}\cos \theta \\ \sin \theta\end{array}\right)=\left(\begin{array}{l}c_{31} \\ c_{32}\end{array}\right)$

$\Rightarrow\left\{\begin{array}{l}s\left(\cos \theta \cdot c_{21}-\sin \theta \cdot c_{22}\right)-t \cos \theta=c_{31}-\cos \theta \cdot c_{11}+\sin \theta \cdot c_{12} \\ s\left(\sin \theta \cdot c_{21}+\cos \theta \cdot c_{22}\right)-t \sin \theta=c_{32}-\sin \theta \cdot c_{11}-\cos \theta \cdot c_{12}\end{array}\right.$

$\Rightarrow-c_{22} t=\sin \theta \cdot\left(c_{21} c_{31}+c_{22} c_{32}\right)+\cos \theta \cdot\left(c_{22} C_{31}-c_{21} c_{32}\right)-c_{11} c_{22}+c_{21} c_{12}$

Barring the degenerate case of $c_{22}=0$ which implies $\mathrm{e}_{\mathrm{jiy}}=0$ and hence $\mathbf{e}_{\mathrm{j}} / \mathbf{e}_{\mathrm{i}}$, the last equation becomes

$t=a \sin \theta+b \cos \theta+d=\sqrt{a^{2}+b^{2}} \sin (\theta+\beta)+d=c \sin (\theta+\beta)+d$

where $\beta, a, b, c$, and $d$ are constants determined by the geometry of $\mathrm{A}, \mathrm{P}_{\mathrm{m}}$ and $\mathrm{P}_{\mathrm{n}}$. The above equation introduces a critical curve in the parameter domain $(t, \theta)$ whose image on the parameterized ruled surface (a helicoid in this case) is the parameterized critical curve attributed to two E-V type contact pairs. It can be seen that the parametric forms of critical curves $\alpha$ and $\beta$ are identical and thus can be regarded as the dual type of each other.

\section{Critical Curve $\gamma$ with E-V and V-E Contact Pairs}

Finally, we consider the case in which the critical curve is a mixture of the two previous curves, which results from two contact pairs $<e_{i}, b_{m}>$ and $<a_{j}, E_{n}>$, where $e_{i}$ and $a_{j}$ are an edge and a vertex of $A$, respectively, and $b_{m}$ and $E_{n}$ are a vertex and an edge of $P_{m} \in \Xi$ and $P_{n} \in \Xi$, respectively, $P_{m} \neq P_{n}$.

As illustrated in Figure A-4, we assign the local coordinate frame $\left\{\mathbf{o}_{\mathrm{e}}, \mathbf{e}_{\mathrm{x}}, \mathbf{e}_{\mathrm{y}}\right\}$ by $\mathbf{o}_{\mathrm{e}}=\mathbf{a}_{\mathrm{i}}, \mathbf{e}_{\mathrm{x}}=\mathbf{e}_{\mathrm{i}}, \mathbf{e}_{\mathrm{y}} \perp \mathbf{e}_{\mathrm{i}}$, where $\mathbf{a}_{\mathrm{i}}$ is the vertex of $A$ incident to $\mathbf{e}_{i}, b_{m}$ is a vertex of $P_{m}$ and $E_{n}$ is an edge of $P_{n}$. The normalized direction along $\mathrm{e}_{\mathrm{i}}$ and $\mathrm{E}_{\mathrm{n}}$ are denoted by $\mathbf{e}_{\mathrm{i}}$ and $\mathbf{E}_{\mathrm{n}}$, respectively. Denoting the position of point $\mathbf{a}_{\mathrm{j}}$ in $\left\{\mathbf{o}_{\mathrm{e}}, \mathbf{e}_{\mathrm{x}}, \mathbf{e}_{\mathrm{y}}\right\}$ as 
$\mathbf{a}_{\mathrm{ji}}$, and parameterizing the placement of $\mathrm{A}$ by $(\mathrm{t}, \theta)$, we have

$$
\begin{gathered}
\mathbf{a}_{i}=\mathbf{b}_{m}-\left(\begin{array}{l}
t \cos \theta \\
t \sin \theta
\end{array}\right) \Rightarrow \mathbf{a}_{i}=\mathbf{b}_{m}-T(t, \theta) \\
\mathbf{a}_{j}=H\left(\mathbf{a}_{j i}\right)=\mathbf{R}(\theta) \mathbf{a}_{j i}+\mathbf{a}_{i}=\mathbf{R}(\theta) \mathbf{a}_{j i}+\mathbf{b}_{m}-T(t, \theta) \\
\mathbf{a}_{j}=\mathbf{b}_{n}+s \mathbf{E}_{n}=\mathbf{R}(\theta) \mathbf{a}_{j i}+\mathbf{b}_{m}-T(t, \theta) \\
\Rightarrow \mathbf{R}(\theta) \mathbf{a}_{j i}-s \mathbf{E}_{n}-T(t, \theta)=\mathbf{b}_{n}-\mathbf{b}_{m} \\
\Rightarrow \mathbf{R}(\theta) \mathbf{c}_{1}-s \mathbf{E}_{n}-T(t, \theta)=\mathbf{c}_{3} \\
\Rightarrow \quad \mathbf{R}(\theta) \mathbf{c}_{1}-s \mathbf{E}_{n}-T(t, \theta)=\mathbf{c}_{3} \\
\Rightarrow\left(\begin{array}{ll}
\cos \theta & -\sin \theta \\
\sin \theta & \cos \theta
\end{array}\right)\left(\begin{array}{l}
c_{11} \\
c_{12}
\end{array}\right)-s\left(\begin{array}{l}
E_{n 1} \\
E_{n 2}
\end{array}\right)-t\left(\begin{array}{l}
\cos \theta \\
\sin \theta
\end{array}\right)=\left(\begin{array}{l}
c_{31} \\
c_{32}
\end{array}\right) \\
\Rightarrow\left\{\begin{array}{l}
\cos \theta \cdot c_{11}-\sin \theta \cdot c_{12}-s E_{n 1}-t \cos \theta=c_{31} \\
\sin \theta \cdot c_{11}+\cos \theta \cdot c_{12}-s E_{n 2}-t \sin \theta=c_{32}
\end{array} \Rightarrow\right.
\end{gathered}
$$

$t\left(\sin \theta \cdot E_{n 1}-\cos \theta \cdot E_{n 2}\right)=c_{31} E_{n 2}-c_{32} E_{n 1}-$

$$
c_{11}\left(\cos \theta \cdot E_{n 2}-\sin \theta \cdot E_{n 1}\right)+c_{12}\left(\cos \theta \cdot E_{n 1}+\sin \theta \cdot E_{n 2}\right)
$$

Ignoring the degenerate case of $\sin \theta \cdot \mathrm{E}_{\mathrm{n} 1}-\cos \theta \cdot \mathrm{E}_{\mathrm{n} 2}=0$ (which means $\mathbf{e}_{\mathrm{i}} / / \mathbf{E}_{\mathrm{n}}$ ), let

$$
\cos \gamma=\frac{E_{n 1}}{\sqrt{E_{n 1}^{2}+E_{n 2}^{2}}}, \quad \sin \gamma=\frac{E_{n 2}}{\sqrt{E_{n 1}^{2}+E_{n 2}^{2}}}
$$

we have:

$\sin \theta \cdot E_{n 1}-\cos \theta \cdot E_{n 2}=\sqrt{E_{n 1}^{2}+E_{n 2}^{2}}(\sin \theta \cdot \cos \gamma-\cos \theta \cdot \sin \gamma)$ $=\sqrt{E_{n 1}^{2}+E_{n 2}^{2}} \sin (\theta-\gamma)$

and

$\cos \theta \cdot E_{n 1}+\sin \theta \cdot E_{n 2}=\sqrt{E_{n 1}^{2}+E_{n 2}^{2}}(\cos \theta \cdot \cos \gamma+\sin \theta \cdot \sin \gamma)$

$=\sqrt{E_{n 1}^{2}+E_{n 2}^{2}} \cos (\theta-\gamma)$

thus

$t\left(\sin \theta \cdot E_{n 1}-\cos \theta \cdot E_{n 2}\right)=$

$d+c_{11}\left(\sin \theta \cdot E_{n 1}-\cos \theta \cdot E_{n 2}\right)+c_{12}\left(\cos \theta \cdot E_{n 1}+\sin \theta \cdot E_{n 2}\right)$

$\Rightarrow t=\frac{d}{\sin \theta \cdot E_{n 1}-\cos \theta \cdot E_{n 2}}+c_{11}+c_{12} \frac{\cos \theta \cdot E_{n 1}+\sin \theta \cdot E_{n 2}}{\sin \theta \cdot E_{n 1}-\cos \theta \cdot E_{n 2}}$

$\Rightarrow t=\frac{p}{\sin (\theta-\gamma)}+r \frac{\cos (\theta-\gamma)}{\sin (\theta-\gamma)}+q$

where $\gamma, p, q$ and $r$ are all constants determined by the geometry of $\mathrm{A}, \mathrm{P}_{\mathrm{m}}$ and $\mathrm{P}_{\mathrm{n}}$.

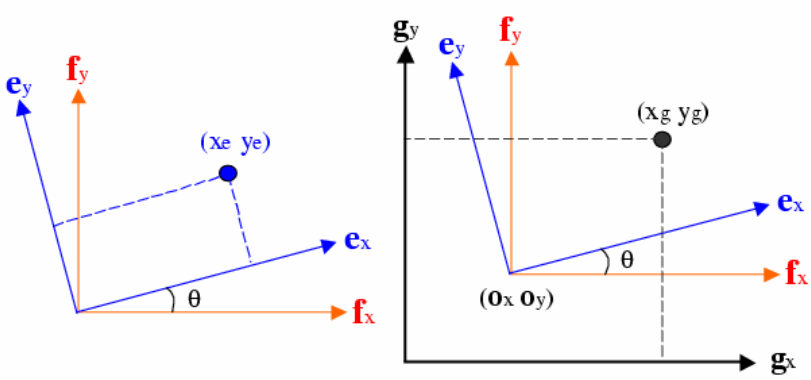

Fig. A-1. Coordinate transformation

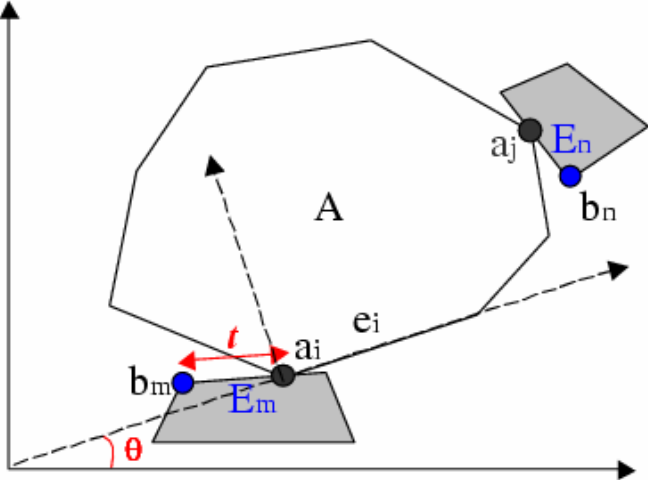

Fig. A-2. V-E and V-E contact critical curve

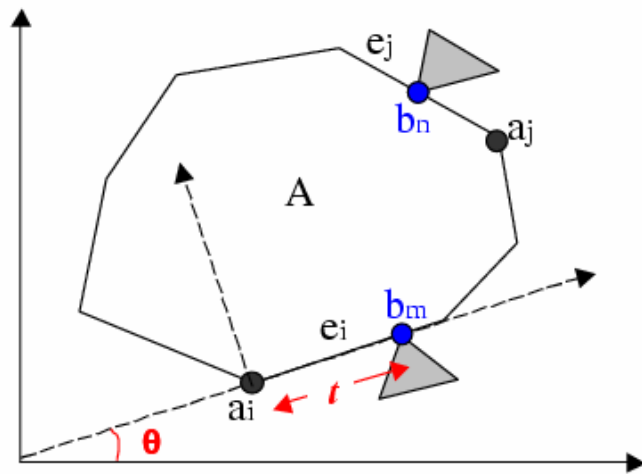

Fig. A-3. E-V and E-V contact critical curve

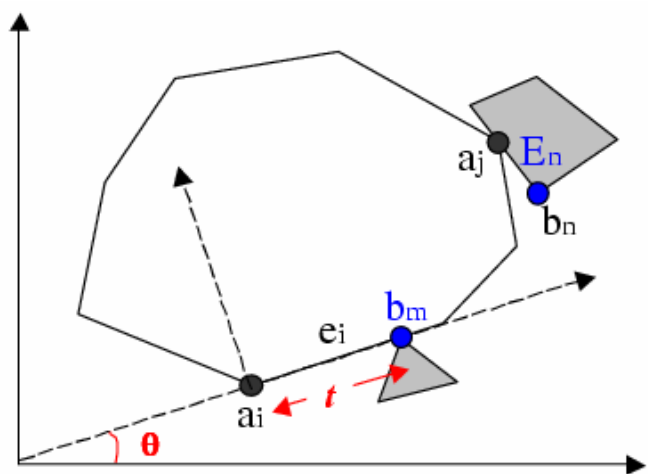

Fig. A-4. E-V and V-E contact critical curve

\section{REFERENCES}

[1] P. K. Agarwal, B. Aronov, M. Sharir, "Motion planning for a convex polygon in a polygonal environment," Discrete Comput. Geom., vol. 22, pp. 201-221, 1999.

[2] D. Z. Chen, X. S. Hu, X. D. Wu, "Optimal polygon cover problems and applications," International Journal of Computational Geometry \& Applications, 12(4):309-338, 2002.

[3] L. P. Chew, K Kedem, "A convex polygon among polygonal obstacles: placement and high-clearance motion," Comput. Geom. Theory Appl., 3(2):59-89, 1993.

[4] R. Church, C. ReVelle, "The maximal covering location problem," Papers of the Regional Science Association, 32, 101-118, 1974.

[5] T. H. Cormen, C. E. Leiserson, R. L. Rivest, Introduction to algorithms. $2^{\text {nd }}$ ed, Cambridge, Mass. : MIT Press ; Boston : McGraw-Hill, 2001.

[6] B. DasGupta, V. P. Roychowdhury, "Two Geometric Optimization Problems," in Ding-Zhu Du and Jie Sun (eds.), New Advances in 
Optimization and Approximation, Kluwer Academic Publishers, pp. 30-57, 1994.

[7] M. de Berg, M. J. Katz, M. H. Overmars, A. F. van der Stappen, J Vleugels, "Models and motion planning," Comput. Geom. Theory Appl., vol. 23, pp. 53-68, 2002.

[8] M. P. do Carmo, Differential geometry of curves and surfaces. Englewood Cliffs, N.J. : Prentice-Hall, 1976.

[9] H. Edelsbrunner, S. S. Skiena, "Probing convex polygons with X-rays," SIAM Journal on Computing, 17(5):870-82, 1988.

[10] Q. J. Ge, J. M. McCarthy, "An algebraic formulation of configuration-space obstacles for spatial robots," in Proceedings 1990 IEEE International Conf. on Robotics \& Automation, vol 3, 1542-7, 1990.

[11] R. B. Grinde, K. Daniels, "Solving an apparel trim placement problem using a maximum cover problem approach," IIE Transactions, 31(8): 763-9, 1999.

[12] K. Kedem, M. Sharir, "An efficient motion planning algorithm for a convex rigid polygonal object in 2-dimentional polygonal case," Discrete Comput. Geom., vol. 5, pp. 43-75, 1990.

[13] K. Kedem, M. Sharir, B. Toledo, "On critical orientations in the Kedem-Sharir motion planning algorithm for a convex polygon in the plane," Discrete Comput. Geom., vol. 17, pp. 227-239, 1997.

[14] J. C. Latombe, Robot motion planning. Kluwer Academic Publishers, 1991.

[15] J. D. Lawrence, A catalog of special plane curves. New York : Dover Publications, 1972.

[16] D. Leven, M. Sharir, "On the number of critical free contacts of a convex polygonal object moving in two-dimensional polygonal space," Discrete Comput. Geom., vol. 2, pp. 255-170, 1987.

[17] T. Lozona-Perez, M. A. Wesley, "An algorithm for planning collision-free paths among polyhedral obstacles," Comm. ACM, 22(10):560-70, 1979.

[18] J. Majhi, R. Janardan, M. Smid, P. Gupta, "On some geometric optimization problems in layered manufacturing," Comput. Geom. Theory Appl., vol. 12, pp. 219-239, 1999.

[19] J. Majhi, R. Janardan, M. Smid, P. Gupta, "Minimizing support structures and trapped area in two-dimensional layered manufacturing," Comput. Geom. Theory Appl., vol. 12, pp. 241-267, 1999.

[20] D. M. Mount, "Packing and covering the plane with translates of a convex polygon," Journal of Algorithms, 11(4):564-580, 1990.

[21] D. M. Mount, R. Silverman, A. Y. Wu, "On the area of overlap of translated polygons," Computer Vision \& Image Understanding, 64(1): 53-61, 1996.

[22] M. Sharir, P. K. Agarwal, Davenport-Schinzel sequences and their geometric applications. Cambridge University Press, 1995.

[23] M. Sharir, S. Toledo S, "Extremal polygon containment problems," Comput. Geom. Theory Appl., 4, 99-118, 1994.

[24] J. T. Schwartz, M. Sharir, J. Hopcroft, Planning, geometry, and complexity of robot motion. Norwood, N.J. : Ablex Pub. Corp., 1987.

[25] E. V. Shikin, Handbook and atlas of curves. Boca Raton : CRC Press, 1995.

[26] K. Tang, T. Woo, J. Gan, "Maximum intersection of spherical polygons and workpiece orientation for 4- and 5-axis machining," ASME Journal of Mechanical Design, vol. 114, pp. 477-485, 1992.

[27] K. Tang, "On computing contact configurations of a curved chain," Graphical Models and Image Processing, vol. 61, pp. 341-361, 1999. and Technology. He received his PhD (2003) and MPhil (1999) in Mechanical Engineering at HKUST, and received his B.Eng. (1998) in Mechano-Electronic Engineering, Tianjin University, P. R. China. His research interests include geometric modeling, computer graphics and design automation.

Kai Tang is currently a faculty member in the Department of Mechanical Engineering at Hong Kong University of Science and Technology. Before joining HKUST in 2001, he had worked for more than 13 years in the $\mathrm{CAD} / \mathrm{CAM}$ and IT industries. His research interests concentrate on designing efficient and practical algorithms for solving real world computational, geometric, and numerical problems. Dr. Tang received his $\mathrm{PhD}$ in Computer Engineering from the University of Michigan in 1990, MSc in Information and Control Engineering in 1986 also from the University of Michigan, and BSc in Mechanical Engineering from Nanjing Institute of Technology in China in 1982.

Yong-Jin Liu is currently a research associate in the Department of Industrial Engineering and Engineering Management, Hong Kong University of Science 